FASTPCR software for PCR, in silico PCR, and oligonucleotide assembly and analysis

\title{
Kalendar, Ruslan
}

Humana press

2014

Kalendar , R , Lee , D \& Schulman , A 2014 , FASTPCR software for PCR, in silico PCR, and oligonucleotide assembly and analysis . in S Valla \& R Lale (eds), DNA Cloning and Assembly Methods . vol. 1116 , Methods in Molecular Biology, vol. 1116 , Humana press, Springer Science+Business Media, New York , pp. 271-302 . https://doi.org/10.1007/978-1-62703-764-8_18

http://hdl.handle.net/10138/155666

https://doi.org/10.1007/978-1-62703-764-8_18

unspecified

submittedVersion

Downloaded from Helda, University of Helsinki institutional repository.

This is an electronic reprint of the original article.

This reprint may differ from the original in pagination and typographic detail.

Please cite the original version. 


\section{Metadata of the chapter that will be visualized online}

Chapter Title $\quad$ FastPCR Software for PCR, In Silico PCR, and Oligonucleotide

Assembly and Analysis

\begin{tabular}{lll}
\hline Copyright Year & 2014 & \\
\hline Copyright Holder & Springer Science+Business Media, New York \\
\hline Corresponding Author & Family Name & Kalendar \\
& Particle & \\
& Given Name & Ruslan \\
& Suffix & \\
& Division & MTT/BI Plant Genomics Laboratory \\
& Organization & Institute of Biotechnology, University \\
& of Helsinki \\
& Address & 65, 00014, Helsinki, Finland \\
& Organization & PrimerDigital Ltd \\
& Address & 00790, Helsinki, Finland \\
\hline Author & Family Name & Lee \\
& Particle & \\
Given Name & Daffix & \\
\hline
\end{tabular}

Author

Family Name

Schulman

Particle

Given Name

Alan H.

Suffix

Abstract

This chapter introduces the software FastPCR as an integrated tools environment for PCR primer and probe design. It also predicts oligonucleotide properties based on experimental studies of PCR efficiency. The software provides comprehensive facilities for designing primers for most PCR applications and their combinations, including standard, multiplex, long-distance, inverse, real-time, group-specific, unique, and overlap extension PCR for multi-fragment assembly in cloning, as well as bisulphite modification assays. It includes a program to design oligonucleotide sets for long sequence assembly by the ligase chain reaction. The in silico PCR primer or probe search includes comprehensive analyses of individual primers and primer pairs. It calculates the melting temperature for standard and degenerate oligonucleotides including LNA and other modifications, provides analyses for a set of primers with prediction of oligonucleotide properties, dimer and G/C-quadruplex detection, and linguistic complexity, and provides a dilution and resuspension calculator. The program includes various bioinformatics tools for analysis of sequences with CG or AT skew, of CG content and purine-pyrimidine skew, and of linguistic sequence complexity. It also permits generation of 
Author's Proof

random DNA sequence and analysis of restriction enzymes of all types. It finds or creates restriction enzyme recognition sites for coding sequences and supports the clustering of sequences. It generates consensus sequences and analyzes sequence conservation. It performs efficient and complete detection of various repeat types and displays them. FastPCR allows for sequence file batch processing, which is essential for automation. The FastPCR software is available for download at http://primerdigital.com/fastpcr.html and online version at http://primerdigital.com/tools/pcr.html.

Keywords

(separated by “-”)
PCR primer design - Primer linguistic complexity - Sequence assembly - Software probe design - Ligase chain reaction - DNA primers 


\section{Author's Proof}

\section{Chapter 18}

\section{FastPCR Software for PCR, In Silico PCR, and Oligonucleotide Assembly and Analysis}

\author{
Ruslan Kalendar, David Lee, and Alan H. Schulman
}

Key words PCR primer design, Primer linguistic complexity, Sequence assembly, Software probe design, Ligase chain reaction, DNA primers

\begin{abstract}
This chapter introduces the software FastPCR as an integrated tools environment for PCR primer and 6 probe design. It also predicts oligonucleotide properties based on experimental studies of PCR efficiency. 7 The software provides comprehensive facilities for designing primers for most PCR applications and their 8 combinations, including standard, multiplex, long-distance, inverse, real-time, group-specific, unique, and overlap extension PCR for multi-fragment assembly in cloning, as well as bisulphite modification assays. It includes a program to design oligonucleotide sets for long sequence assembly by the ligase chain reaction. The in silico PCR primer or probe search includes comprehensive analyses of individual primers and primer pairs. It calculates the melting temperature for standard and degenerate oligonucleotides including LNA and other modifications, provides analyses for a set of primers with prediction of oligonucleotide properties, dimer and G/C-quadruplex detection, and linguistic complexity, and provides a dilution and resuspension calculator. The program includes various bioinformatics tools for analysis of sequences with $\mathrm{CG}$ or AT skew, of CG content and purine-pyrimidine skew, and of linguistic sequence complexity. It also permits generation of random DNA sequence and analysis of restriction enzymes of all types. It finds or creates restriction enzyme recognition sites for coding sequences and supports the clustering of sequences. It generates consensus sequences and analyzes sequence conservation. It performs efficient and complete detection of various repeat types and displays them. FastPCR allows for sequence file batch processing, which is essential for automation. The FastPCR software is available for download at http://primerdigital.com/ fastpcr.html and online version at http://primerdigital.com/tools/pcr.html.

Abstract
\end{abstract}

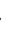
8 9

\section{Abbreviation}

OE-PCR Overlap extension PCR

PCR

Polymerase chain reaction

RT-PCR Real-time PCR

SSR

Simple sequence repeat 


\section{Author's Proof}

The polymerase chain reaction (PCR) is fundamental to molecular biology and is the most important practical molecular technique for the DNA research laboratory. However, the utility of the method is dependent on identifying unique primer sequences and designing PCR-efficient primers. Primer design is a critical step in all types of PCR methods to ensure specific and efficient amplification of a target sequence [1-7]. Even though there are currently many online and commercial bioinformatics tools, primer design for PCR is still not as convenient and practical as it might be for routine use. The adaptation of PCR for different applications has made it necessary to develop new criteria for PCR primer and probe design to cover uses such as RT-PCR, real-time PCR, groupspecific and unique PCR, combinations of multiple primers in multiplex PCR, overlap extension PCR fort multi-fragments assembling cloning, and bisulphite modification assays. There is a need as well as for a program integrating design oligonucleotide sets for long sequence assembly by the ligase chain reaction (LCR), discovery of simple sequence repeats (SSRs) and their amplification as diagnostic markers, and for designing TaqMan, molecular beacon, and microarray oligonucleotides $[6,8,9]$.

In developing FastPCR and Java web tools (Table 1), our aim was to create practical and easy-to-use software for routine manipulation and analysis of sequences for most PCR applications. The parameters adopted are based on our experimental data for efficient PCR and are translated into algorithms in order to design combinations of primer pairs for optimal amplification. This software, FastPCR, has been successfully used throughout the scientific community in a wide range of PCR and probe applications, and repeat searches and analysis. The first papers describing this software were published in 2001 and since 1999 this software has been applied in various projects, have been cited together over 500 times in scientific journals, patents, $\mathrm{PhD}$ theses, and over 1,000 downloads of the installation file per month. The program code is regularly updated.

\section{Software, General Information}

The FastPCR software (http://primerdigital.com/fastpcr.html) is written in Microsoft Visual Studio 6.0 and compiled to an executive file that, after installation, can be used with any version of Microsoft Windows. For Linux and Mac it requires "Wine" (http://www.winehq.org/) as a compatibility layer for running Windows programs. It is a completely free alternative implementation of the Windows API also for use with native Windows DLLs. 


\section{Author's Proof}

PCR Primer and Probe Design and Oligonucleotide Assembly and Analysis

t1.1 Table 1

t1.2 Summary of the FastPCR software for PCR, in silico PCR, and oligonucleotide assembly and analysis

$\mathrm{t} 1.3$

$\mathrm{t} 1.4$

$\mathrm{t} 1.5$

$\mathrm{t} 1.6$

$\mathrm{t} 1.7$

$\mathrm{t} 1.8$

$\mathrm{t} 1.9$

t1.10

$\mathrm{t} 1.11$

t1.12

t1.13

t1.14

t1.15

t1.16

$\mathrm{t} 1.17$

t1.18

t1.19

t1.20

$\mathrm{t} 1.21$

t1.22

$\mathrm{t} 1.23$

$\mathrm{t} 1.24$

t1.25

$\mathrm{t} 1.26$

$\mathrm{t} 1.27$

$\mathrm{t} 1.28$

t1.29

$\mathrm{t} 1.30$

$\mathrm{t} 1.31$

t1.32

\section{Features}

PCR tool provides comprehensive facilities for

Design of primers for most PCR applications and their combinations, including standard, multiplex, long-distance, inverse, real-time, unique (specific primers for each from genetically related DNA sequences) or group-specific (universal primers for genetically related DNA sequences), linear-afterthe-exponential (LATE)-PCR, bisulphite modification assays, polymerase extension PCR multifragment assembly cloning

Design of long oligonucleotides for microarray analyses and dual-labeled oligonucleotides for probes such as molecular beacons

Polymerase chain assembly (PCA) or oligo assembly—for automating the design of oligonucleotide sets for long sequence assembly by ligase chain reaction (LCR) and PCR

In silico (virtual) PCR or multiple primer or probe searches, or in silico PCR against whole genome(s) or a list of predictions by chromosome of probable PCR products, and search for potential mismatching locations of the specified primers or probes

Testing of individual primers, melting temperature calculation for standard and degenerate oligonucleotides including LNA and other modifications

Evaluation of PCR efficiency, linguistic complexity, dimer and G/C-quadruplex detection, dilution and resuspension calculator

Analysis of features of multiple primers simultaneously, including $T_{\mathrm{m}}$, CG content, linguistic complexity, dimer formation; optimal $T_{a}$

Identification of simple sequence repeat (SSR) loci by analyzing the low-complexity regions of input sequences

Restriction digest analyses for Type I, II, and III restriction enzymes and homing endonucleases, finding or creating restriction enzyme recognition sites for coding sequences

Searches for similar sequences (or primers)

Translation of nucleotide (DNA/RNA) sequences to the corresponding peptide sequence in all six frames for standard and degenerate DNA and modifications (inosine, uridine)

Determination of CG: $(\mathrm{G}-\mathrm{C}) /(\mathrm{G}+\mathrm{C}), \mathrm{AT}:(\mathrm{A}-\mathrm{T}) /(\mathrm{A}+\mathrm{T}), \mathrm{SW}:(\mathrm{S}-\mathrm{W}) /(\mathrm{S}+\mathrm{W}), \mathrm{MK}:(\mathrm{M}-\mathrm{K}) /(\mathrm{M}+\mathrm{K})$, purine-pyrimidine $(\mathrm{R}-\mathrm{Y}) /(\mathrm{R}+\mathrm{Y})$ skews, $\mathrm{CG} \%$ content and the melting temperature, primer quality and linguistic sequence complexity profiles

The online FastPCR (jPCR) software (http://primerdigital.com/ tools/) is written in Java with NetBeans IDE (Oracle) and requires 75 the Java Runtime Environment (JRE) on a computer. It can be 76 used with any operating system (64-bit OS preferred for large 77 chromosome files). 


\section{Author's Proof}

Ruslan Kalendar et al.

\section{The Interface}

\subsection{Inputs to FastPCR}

The software contains the menus, the toolbars, and the ribbon and three text editors. The ribbon is designed to help the user quickly find the commands that are needed to complete a task. Commands are organized in logical groups, which are collected together under tabs (Fig. 1). Each tab relates to a type of activity, such as "PCR Primer Design," “in silico PCR," or "Primer Test."

Getting started with a basic project in FastPCR software is as easy as opening a new or existing file than using copy-paste or starting to type. There are three independent text editors on different tabs within the interface: "General sequence(s)," "Additional sequence(s) or pre-designed primers (probes) list," and "Result report." The first two text editors are necessary for loading sequences for analysis, the text editor "General sequence(s)" is designed for working with the project sequences, and the "Additional sequence(s) or pre-designed primers (probes) list" text editor is used for special and additional sequences such as predesigned primers, multiple query sequences, or numbers for input.

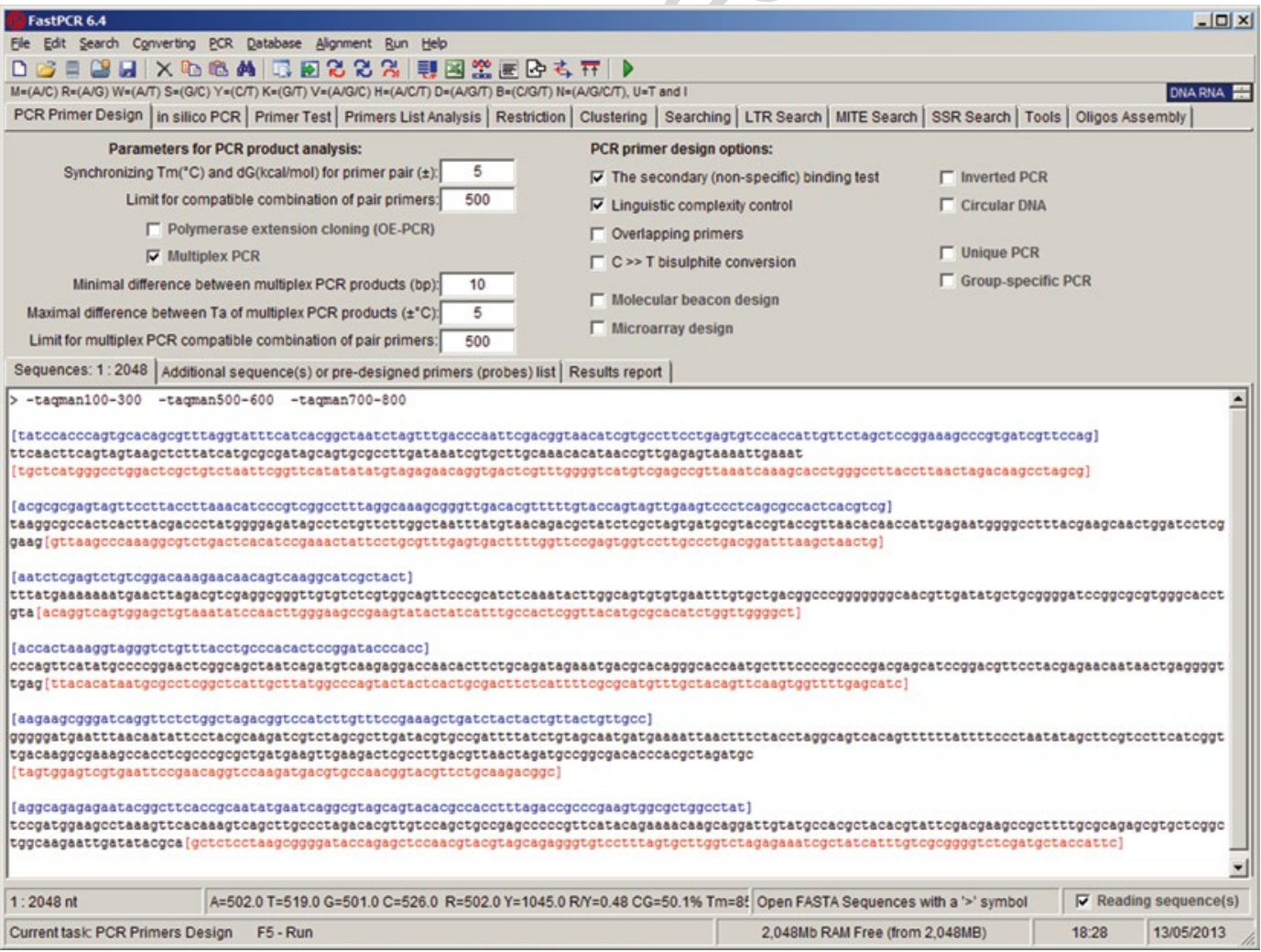

Fig. 1 The FastPCR sequence editor and user interface 


\section{Author's Proof}

\subsection{Program Output}

\subsection{Sequence Entry}

3.4 FASTA Format Description
FastPCR automatically generates results at a third text editor, "Result report," in tabulated format for transferring to spreadsheet software from the clipboard using copy-paste. Output results are easy to save as Excel worksheet (.xls) or as Rich Text Format (.rtf) text files, compatible with MS Excel or Open Office. The separated output of the primer design is a list of primers, a set of primer pair sequences with their theoretical PCR products, and, for multiplex PCR, the result of the calculation of multiple-PCR primers for given target sequences. In addition, the output shows optimal annealing temperature for each primer pair, the size of PCR product, and complete information for each designed primer and for each multiplex PCR product set.

Sequence data files are prepared using a text editor (Notepad, 109 WordPad, MS Word), and saved in ASCII as text/plain format (.txt) 110 or in .rtf. The program either takes a single sequence or accepts 111 multiple separate DNA sequences in FASTA, tabulated format (two 112 columns from MS Excel sheet or MS Word table), EMBL, MEGA, 113 GenBank, MSF, DIALIGN, simple alignment, or BLAST Queue 114 web alignment formats. The template length is not limited. The 115 FastPCR clipboard allows the user to copy and paste text or tables 116 from MS Word documents or MS Excel worksheets or other programs 117 and to paste them into another Office document. It is important that 118 all target sequences are prepared in the same format. Users can type 119 or import from file(s) into "General Sequence(s)" or "Additional 120 sequence(s) or pre-designed primers (probes) list" editors. 121

FastPCR allows files to be opened in several ways: the original 122 file can be opened as read-only for editing with text editors; files 123 can be opened to memory without using text editors, which allows 124 larger file(s), up to $200 \mathrm{Mb}$, to be analyzed; files within a folder can 125 be selected and the files opened during task execution without the 126 use of text editor program. Additionally, the program can open 127 files within a selected folder in order to join all these files in a text 128 editor. For example, this feature can be applied to convert all files 129 from a selected folder into a single file of FASTA sequences. 130 Alternatively this feature allows splitting FASTA sequences to indi- 131 vidual files in a particular selected folder.

When a sequence file is opened, FastPCR displays the information about the opened sequence and its format. The information status bar shows the number of sequences, the total sequence length (in nucleotides), the nucleotide composition, and the purine, pyrimidine, CG percentage, and the melting temperature. Files can be saved in various formats including .rtf, .xls, or txt from the text editor in use.

FastPCR normally expects to read sequence files in FASTA format [10]. FASTA format has the highest priority and is simple, because the raw sequence is merely preceded by a definition line. The definition line begins with a ">" sign and optionally followed 00

101

102

103

104

105

106

107

108

9

1

2

14

5

7

19

1

23

5

6

28

9

13




\section{Author's Proof}

Ruslan Kalendar et al.

immediately by a name for the sequence with no length restriction. Many sequences can be listed in the file, the format indicating a new sequence at each "> "symbol found. It is important to press Enter at the end of each line after ">" to help FastPCR recognize the end and beginning of the sequence and the sequence name. It is important that the first line of each sequence starts with ">."

Degenerate DNA sequences are accepted as IUPAC code [11], an extended vocabulary of 11 letters, which allows the description of ambiguous DNA code. Each letter represents a combination of one or several nucleotides: $\mathrm{M}=(\mathrm{A} / \mathrm{C}), \mathrm{R}=(\mathrm{A} / \mathrm{G}), \mathrm{W}=(\mathrm{A} / \mathrm{T})$, $\mathrm{S}=(\mathrm{G} / \mathrm{C}), \mathrm{Y}=(\mathrm{C} / \mathrm{T}), \mathrm{K}=(\mathrm{G} / \mathrm{T}), \mathrm{V}=(\mathrm{A} / \mathrm{G} / \mathrm{C}), \mathrm{H}=(\mathrm{A} / \mathrm{C} / \mathrm{T})$, $\mathrm{D}=(\mathrm{A} / \mathrm{G} / \mathrm{T}), \quad \mathrm{B}=(\mathrm{C} / \mathrm{G} / \mathrm{T}), \quad \mathrm{N}=(\mathrm{A} / \mathrm{G} / \mathrm{C} / \mathrm{T}), \mathrm{U}=\mathrm{T}$, and $\mathrm{I}$ (Inosine). The program accepts amino acid codes: A (Ala), C (Cys), D (Asp), E (Glu), F (Phe), G (Cly), H (His), I (Ile), K (Lys), L (Leu), M (Met), N (Asn), P (Pro), Q (Gln), R (Arg), S (Ser), T (Thr), U (Sec), V (Val), W (Trp), and Y (Tyr).

\subsection{Alignment Format Description}

\section{The PCR Primers or Probe Design Analysis Options}

\subsection{PCR Primer}

\section{Design Generalities}

There are many different programs that perform different types of alignment formats. Standardizing on a set of formats enables programs to be written that can read results from many different programs. In all alignment formats, gaps that have been introduced into the sequences to make them align are indicated by the "-" character. The exception to this rule is GCG/MSF format which uses "." as the gap character inside the sequences. The file may begin with as many lines of comment or description as required. The first mandatory line must contain the text "MSF," "Alignment as simple alignment format," "DIALIGN," or "MEGA" to be recognized as alignments from these programs. Following the first line are lines that start with the sequence name, which is separated from the aligned sequence residues by white space.
Primer design is one of the key steps for successful PCR. For PCR applications, primers are usually $18-35$ bases in length and should be designed such that they have complete sequence identity to the desired target fragment to be amplified. The parameters, either controllable by the user or selected automatically, are primer length (12-500 nt), melting temperature for short primers calculated by nearest neighbor thermodynamic parameters, theoretical primer PCR efficiency (quality at \%) value, primer CG content, $3^{\prime}$ end terminal enforcement, preferable $3^{\prime}$ terminal nucleotide sequence composition in degenerated formulae, and added sequence tags at $5^{\prime}$ termini. The other main parameters used for primer selection are the general nucleotide structure of the primer such as linguistic complexity (nucleotide arrangement and composition), specificity, 


\section{Author's Proof}

PCR Primer and Probe Design and Oligonucleotide Assembly and Analysis

$\begin{array}{ll}\text { t2.1 } & \text { Table } 2 \\ \text { t2.2 } & \text { Default primer design selection criteria }\end{array}$

\begin{tabular}{|c|c|c|}
\hline Criteria & Default & Ideal \\
\hline Length (nt) & $20-24$ & $>21$ \\
\hline$T_{\mathrm{m}}$ range $\left({ }^{\circ} \mathrm{C}\right)^{\mathrm{a}}$ & $52-68$ & $60-68$ \\
\hline$T_{m}{ }^{a} 12$ bases at $3^{\prime}$ end & $30-50$ & $41-47$ \\
\hline CG $(\%)$ & $45-65$ & 50 \\
\hline $3^{\prime}$ end composition $\left(5^{\prime}-\mathrm{NNN}-3^{\prime}\right)^{\mathrm{b}}$ & sWW, SWS, SSW, WSS & SSA, SWS, WSS \\
\hline Sequence linguistic complexity $(L C, \%)^{c}$ & $>75$ & $>90$ \\
\hline Sequence quality $(P Q \%)$ & $>70$ & $>90$ \\
\hline $\begin{array}{l}\text { Nearest neighbor thermodynamic paramete } \\
\text { Ambiguity codes }\end{array}$ & cording to SantaLucia [13] & \\
\hline
\end{tabular}

the melting temperature of the whole primer, the melting 187 temperature at the $3^{\prime}$ and $5^{\prime}$ termini, self-complementarity, and 188 secondary (nonspecific) binding.

The software can dynamically optimize the best primer length for the entered parameters. All PCR primer (probe) design parameters are flexible and changeable according to the specifics of the analyzed SEQUENCES and tasks. Primer pairs are analyzed for cross-hybridization and specificity of both primers and, optionally, selected with similar melting temperatures. Primers with balanced melting temperatures (within $1-6{ }^{\circ} \mathrm{C}$ of each other) are desirable but not mandatory. The default primer design selection criteria are shown in Table 2. It is possible to use predesigned primers or probes or, alternatively, predesigned primers can act as references for the design of new primers. The program accepts a list of predesigned oligonucleotide sequences and checks the compatibility of each primer with a newly designed primer or probe.

\subsection{Melting} Temperature Calculation
The melting temperature $\left(T_{\mathrm{m}}\right)$ is defined as the temperature at 203 which half the DNA strands are in the double-helical state and half 204 are in the "random-coil" state. The $T_{\mathrm{m}}$ for short oligonucleotides 205 with normal or degenerate (mixed) nucleotide combinations is 206 calculated in the default setting using nearest neighbor 207 thermodynamic parameters [12, 13]. The CG content of an 208 oligonucleotide is the most important factor that influences the $T_{\mathrm{m}} \quad 209$ value. The melting temperature for mixed bases is calculated by 210 averaging nearest neighbor thermodynamic parameters-enthalpy 211 and entropy values - at each mixed site; extinction coefficient is 212 similarly predicted by averaging nearest neighbor values at mixed 213 sites $[2,3]$. Mismatched pairs can be taken into account since the 214 parameters provide for DNA/DNA duplexes and dangling ends, 215 


\section{Author's Proof}

Ruslan Kalendar et al.

which are unmatched terminal nucleotides [14-16]. The melting temperature for primer (probe) self- or cross-dimers and for in silico PCR experiments with oligonucleotides having mismatches to the target is calculated using values for the thermodynamic parameters for a nucleic acid duplex.

The FastPCR allows the choice of other nearest neighbor thermodynamic parameters or simple non-thermodynamic $T_{\mathrm{m}}$ calculation formulae. For non-thermodynamic $T_{\mathrm{m}}$ calculation, we suggest using simple formulae; the Wallace-Ikatura rule [17] is often used as a rule of thumb when primer $T_{\mathrm{m}}$ is to be estimated at the bench. However, the formula was originally applied to the hybridization of probes in $1 \mathrm{M} \mathrm{NaCl}$ and is an estimate of the melting temperature for oligonucleotides shorter than 10 bases:

$$
T_{\mathrm{m}}\left({ }^{\circ} \mathrm{C}\right)=2(\mathrm{~L}+\mathrm{G}+\mathrm{C}),
$$

for oligonucleotides longer than ten bases:

$$
T_{\mathrm{m}}\left({ }^{\circ} \mathrm{C}\right)=64.9+\frac{4 \mathrm{l}([\mathrm{G}+\mathrm{C}]-16.4)}{L}
$$

or the formula [18]:

$$
T_{\mathrm{m}}\left({ }^{\circ} \mathrm{C}\right)=77.1+11.7 \log _{10}\left[\mathrm{~K}^{+}\right]+\frac{4 \mathrm{l}[\mathrm{G}+\mathrm{C}]-528}{L}
$$

where $L$ is length of primer, $[\mathrm{G}+\mathrm{C}]$ is the number of Gs and Cs, and $\left[\mathrm{K}^{+}\right]$is salt molar concentration (default value is $50 \mathrm{mM}$ ). The two equations above assume that the stabilizing effects of cations are the same on all base pairs. The melting temperature of the PCR product is calculated using the formula [15]:

$$
T_{\mathrm{m}}\left({ }^{\circ} \mathrm{C}\right)=81.5+16.6 \log _{10}\left[\mathrm{~K}^{+}\right]+\frac{4 \mathrm{l}[\mathrm{G}+\mathrm{C}]-675}{L}
$$

4.3 Linguistic Complexity of Sequence and Nucleotide-Skew Analysis
The sequence complexity calculation method can be used to search for conserved regions between the compared sequences in order to detect low-complexity regions including SSRs, imperfect direct or inverted repeats, polypurine and polypyrimidine triple-stranded DNA structures, and four-stranded structures (such as G/Cquadruplexes) [19]. Linguistic complexity measurements are performed using the alphabet-capacity $L$-gram method $[20,21]$ along the whole sequence length and calculated as the sum of the observed range $\left(x_{i}\right)$, from 1 - to $L$-size words in the sequence, divided by the sum of the expected $(E)$ value for this sequence length. Linguistic complexity $(L C)$ values for sequence length $(s)$ are converted to percentages, in which $100 \%$ means maximal "vocabulary richness" of a sequence: 


\section{Author's Proof}

PCR Primer and Probe Design and Oligonucleotide Assembly and Analysis

$$
L C(\%)=\frac{100 \times \sum_{i=1}^{L} x_{i}}{E},
$$

where

$$
\begin{gathered}
E=\sum_{i=1}^{L}\left\{\frac{s-i+1, s<4^{i}-1+i}{4^{i}, s \geq 4^{i}-1+i},\right. \\
L=\left[\log _{4}\left(\frac{s}{3}\right)+1\right] .
\end{gathered}
$$

For example, the sequence 5'-ACACACACACACACAC, 257 16 nt $(L=3)$, contains two nucleotides (A, C), but expected $E=4258$ variants; two variants of dinucleotides (AC, CA), but expected 259 $E=(16-1)$ variants; two variants of trinucleotides (ACA, CAC), 260 and expected $E=(16-2)$ variants. The complexity value is 261 $\mathrm{LC}=100(2+2+2) /(4+16-1+16-2)=18.2 \%$.

The LC tries to describe the "uniqueness" ("vocabulary richness") of a sequence and the likelihood of PCR success of each primer; this value varies from 100 for the best to 5 (e.g., $\operatorname{poly}(N)$ ) for the worst primers. LC values of 80 and higher allow for the rapid choice of the best primer or probe sequences.

4.4 Primer Quality (Virtual PCR Efficiency) Determination
Our experimental data showed that the primer nucleotide composition and melting temperature of the 12 bases at the $3^{\prime}$ end of the primers are important factors for PCR efficiency. The melting temperature of the 12 bases at the $3^{\prime}$ terminus is calculated preferably by nearest neighbor thermodynamic parameters [13]. The composition of the sequence at the $3^{\prime}$ terminus is important; primers with two terminal $\mathrm{C} / \mathrm{G}$ bases are recommended for increased PCR efficiency [22]. Nucleotide residues C and G form a strong pairing structure in the duplex DNA strands. Stability at the $3^{\prime}$ end in primer template complexes will improve the polymerization efficiency.

We specify an abstract parameter called primer quality (PQ) that can help to estimate the efficiency of primers for PCR. PQ is calculated by the consecutive summation of the points according to the parameters of total sequence and purine-pyrimidine sequence complexity and of the melting temperatures of the whole primer and of the terminal 12 bases at both the $3^{\prime}$ and $5^{\prime}$ ends. Self-complementarity, which gives rise to possible primer-dimer and hairpin structures, reduces the final value. The PQ tries to describe the likelihood of PCR success of each primer; this value varies from 100 for the best to 0 for the worst primers. To meet multiplexing demands, it is possible in the program to select the best primer with an optimal temperature range, allowing the design of qualified primers or probes for any target sequence with any CG 


\section{Author's Proof}

Ruslan Kalendar et al.

and repeat content. PQ values of 80 and higher allow for the rapid choice of the best PCR primer pair combinations. No adverse effects, due to the modification of the reaction buffer, chosen thermostable polymerases, or variations in annealing temperature, have been observed on the reproducibility of PCR amplification using primers with high PQ.

4.5 Hairpin (Loop) and Dimer Formation

Primer-dimers involving one or two sequences may occur in a PCR. The FastPCR tool eliminates intra- and inter-oligonucleotide reactions before generating a primer list and primer pair candidates. It is very important for PCR efficiency that the production of stable and inhibitory dimers is prevented, especially by avoiding complementarity in the $3^{\prime}$ ends of primers from whence the polymerase will extend. Stable primer-dimer formation is very effective at inhibiting PCR because the dimers formed are amplified efficiently and compete with the intended target.

Primer-dimer prediction is based on analysis of non-gapped local alignments and the stability of both the $3^{\prime}$ end and the central regions of the primers (Fig. 2). Primers will be rejected when they have the potential to form stable dimers, depending on the nucleotide composition and with at least five bases at the $3^{\prime}$ end or seven bases in the central region. Tools to calculate $T_{\mathrm{m}}$ for primer-dimers with mismatches for pure, mixed, or modified (inosine, uridine, or locked nucleic acid (LNA)) bases, using averaged nearest neighbor thermodynamic parameters, are provided for DNA/DNA duplexes [12-14, 23, 24].

In addition to Watson-Crick base-pairing, there is a variety of other hydrogen bonding configurations possible [19, 25-27], including G/C-quadruplexes and wobble base pairs, which the FastPCR software detects. The program provides for the detection of alternative hydrogen bonding during primer-dimer and in silico PCR primer binding site detection. The mismatch stability is examined in order of decreasing stability: G-C $>\mathbf{A}-\mathbf{T}>\mathrm{G} \cdot \mathrm{G}>\mathrm{G} \cdot \mathrm{T} \geq$ $\mathrm{G} \cdot \mathrm{A}>\mathrm{T} \cdot \mathrm{T} \geq \mathrm{A} \cdot \mathrm{A}>\mathrm{T} \cdot \mathrm{C} \geq \mathrm{A} \cdot \mathrm{C} \geq \mathrm{C} \cdot \mathrm{C}$. Guanine is the most universal base, because it forms the strongest base pair and the strongest mismatches. However, cytosine is the most discriminating base, because it forms the strongest pair and the three weakest mismatches $[23,28]$. Therefore, the software also looks for stable guanine mismatches: $\mathrm{G} \cdot \mathrm{G}, \mathrm{G} \cdot \mathrm{T}$ and $\mathrm{G} \cdot \mathrm{A}$.

G-rich (and C-rich) nucleic acid sequences can fold into fourstranded DNA structures that contain stacks of G-quartets [19]. These quadruplexes can be formed by the intermolecular association of two or four DNA molecules, dimerization of sequences that contain two $\mathrm{G}$ bases, or by the intermolecular folding of a single strand containing four blocks of guanines. These are easy to eliminate from primer design because of their low linguistic complexity; LC $=32 \%$ for (TTAGGG) 4 $_{4}$. The software predicts the presence of putative G- and C-quadruplexes in primer sequences. 


\section{Author's Proof}

PCR Primer and Probe Design and Oligonucleotide Assembly and Analysis

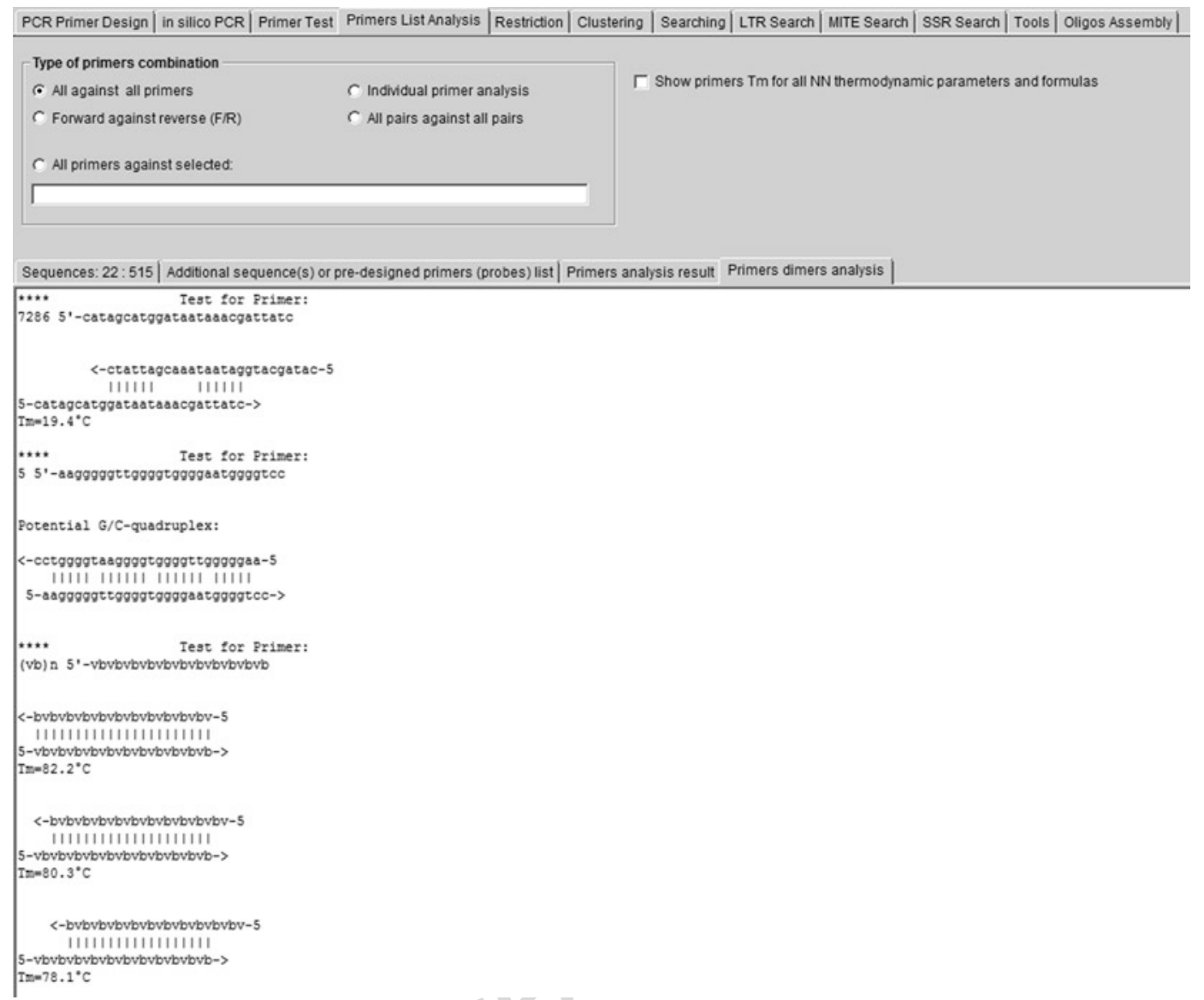

Fig. 2 One of the FastPCR duplex formation results

\subsection{Calculation of Optimal Annealing Temperature}

Intermolecular G-quadruplex-forming sequences are detected 339 according to the formula $\ldots G_{m 1} X_{\mathrm{n}} G_{\mathrm{m} 2} \ldots$, where $m$ is the number 340 of $\mathrm{G}$ residues in each G-tract $\left(m_{1}, m_{2} \geq 3\right)$; the gap $X_{n}\left(n \leq 2{ }^{*}\right.$ mini- 341 mal $\left.\left(m_{1}: m_{2}\right)\right)$ can be any combination of residues, including $\mathrm{G} 342$ [16]. The gap sequences $\left(X_{n}\right)$ may have varying lengths, and a rela- 343 tively stable quadruplex structure may still be formed with a loop 344 more than seven bases long, but in general, increasing the length 345 of the gap leads to a decrease in structure stability. It is also possible 346 for one of the gaps to be zero length when there are long poly-G 347 tracts of $>6$ bases.

The optimal annealing temperature $\left(T_{\mathrm{a}}\right)$ is the temperature, 349 generally stated as a range, where efficiency of PCR amplification is 350 maximal but nonspecific products minimal. The most important 351 values for estimating the $T_{\mathrm{a}}$ are the primer quality, the $T_{\mathrm{m}}$ of the 352 primers, and the length of PCR fragment. Primers with high $T_{\mathrm{m}} 353$ $\left(>60{ }^{\circ} \mathrm{C}\right.$ ) can be used in PCRs with a wide $T_{\mathrm{a}}$ range compared to 354 


\section{Author's Proof}

Ruslan Kalendar et al.

\subsection{The Secondary Nonspecific Binding Test; Alternative Amplification Products}

primers with low $T_{\mathrm{m}}\left(<50{ }^{\circ} \mathrm{C}\right)$. The optimal annealing temperature for PCR is calculated directly as the value for the primer with the lowest $T_{\mathrm{m}}\left(T_{\mathrm{m}}{ }^{\mathrm{min}}\right)$. However, PCR can work in temperatures up to $10^{\circ}$ higher than the $T_{\mathrm{m}}$ of the primer so as to favor primer target duplex formation:

$T_{\mathrm{a}}\left({ }^{\circ} \mathrm{C}\right)=T_{\mathrm{m}}{ }^{\mathrm{min}}+\ln L$, where $L$ is the length of the PCR fragment.

The specificity of the oligonucleotides is one of the most important factors for good PCR; optimal primers should hybridize only to the target sequence, particularly when complex genomic DNA is used as the template. Amplification problems can arise due to primers annealing to repetitious sequences (retrotransposons, DNA transposons, or tandem repeats). Alternative product amplification can also occur when primers are complementary to inverted repeats and produce multiple bands. This is unlikely when primers have been designed using specific DNA sequences (unique PCR). However, the generation of inverted repeat sequences is exploited in two common generic DNA fingerprinting methods, RAPD and AP-PCR [29, 30]. Because only one primer is used in these PCRs, the ends of the products must be reverse complements and thus can form stem-loops.

The techniques of inter-repeats amplified polymorphism: interretrotransposon amplified polymorphism (IRAP), retrotransposonmicrosatellite amplified polymorphism (REMAP), inter-MITE amplification $[31,32]$, and $A l u$-repeat polymorphism $[33,34]$ have exploited highly abundant, dispersed repeats as markers. However, primers complementary to repetitious DNA may produce many nonspecific bands in single-primer amplification and compromise the performance of unique PCRs. A homology search with the primer as the query sequence, for example, using BLASTn against all sequences in GenBank or EMBL-Bank, will determine whether the primer is likely to interact with dispersed repeats. Alternatively, one can create a small, local, specialized library of repeat sequences based on those in Repbase [35] or TREP [36] and use this for searches.

The mismatches at the $3^{\prime}$ end of the primers affect target amplification much more than mismatches at the $5^{\prime}$ end. A twobase mismatch at the $3^{\prime}$ end of the primer prevents amplification. A single-base mismatch at the $3^{\prime}$ end as well as several mismatches at the $5^{\prime}$ end of the primer permits amplification, albeit with reduced efficiency. However, the presence of multiple primer binding sites does not necessarily lead to alternative amplification products because, for successful amplification, the priming sites for both primers must be both located close to each other, in correct orientation, and sufficiently match the primer sequences.

By default, FastPCR performs a test for nonspecific binding by repeats masking and low-complexity regions detection and masking for each given sequence. 
Additionally the software allows this test to be performed against a reference sequence or sequences (e.g., a BAC or YAC) or one's own database. Primers that bind to more than one location on given sequences will be rejected. Even though the test for nonspecific primer binding is performed as a default for all primers, the user may cancel the operation. Identification of secondary binding sites including mismatched hybridization is normally performed by considering the similarity of the primer to targets along the entire primer sequence. An implicit assumption is that stable hybridization of a primer with the template is a prerequisite for priming by DNA polymerase. FastPCR pays particular attention to the $3^{\prime}$ portion of the primers and calculates the similarity of $3^{\prime}$ end to the target (the length is chosen by user) to determine the stability of any potential interactions.

The secondary nonspecific primer binding test is based on repeat masking using a quick local alignment screen (which allows one mismatch within a hash index of 12 -mers) between the reference and input sequences.

Once the input files are selected or sequences copied and pasted to 422 the General Sequence(s) text editor, the FastPCR provides various execution features. Figure 3 provides an example for primer design from the user's perspective.

5.1 Execution of the Selected Task

The user selects the ribbon having the task needed. The program will only perform the selected task. The name of the selected executive task is shown on the status bar by "Press F5." The task is executed by using key $\mathbf{F} \mathbf{5}$ or by clicking the arrow on the toolbar using the mouse. Once the executive task is completed, the result is shown in the Result report text editor (e.g., see Fig. 3).

\subsection{PCR Primer Design Options}

The "PCR Primer Design" Tab contains various execution options for commonly selected types of PCR and for the most important PCR parameters (Fig. 1). The option panel of "PCR Primers or Probe Design Options" is shown in Fig. 4. Once the user selects any attribute, the option attribute value field shows the default attributes value, which can then be modified. "PCR Primers or Probe Design Options" affects all sequences. PCR primer design options can be customized for each sequence using special commands at the header of the sequence (http:// primerdigital.com/soft/pcr_help.html). Typically, it is not necessary to use these commands to manage typical PCR primer design and these are applied to advanced tasks. Default global parameters for primer design will be assigned by typing the help command " ? ?" in text editor: 


\section{Author's Proof}

Ruslan Kalendar et al.

\begin{tabular}{|c|c|c|c|c|c|c|c|c|c|c|c|c|c|}
\hline \multicolumn{14}{|c|}{ Sequences: $3: 844 \mid A$} \\
\hline \multirow{2}{*}{\begin{tabular}{|l} 
PrimerID \\
example1 \\
1F1_1_18-39
\end{tabular}} & \multicolumn{2}{|l|}{ Sequence $\left(5^{\prime}-3^{\prime}\right)$} & \multirow{2}{*}{$\begin{array}{l}\operatorname{Tm}\left({ }^{\circ} \mathrm{C}\right) \\
56.5\end{array}$} & \multicolumn{2}{|c|}{$\mathrm{dG}(\mathrm{kcal} / \mathrm{mol})$} & \multicolumn{2}{|c|}{ Im_3'end ("C) } & \multirow{2}{*}{$\begin{array}{l}C G(3) \\
88\end{array}$} & \multicolumn{4}{|c|}{ Linguistic_Complexity(t) } & \multirow[t]{11}{*}{ Primer_Quality(3) } \\
\hline & tegtattcaggegtacettetg & 22 & & -27.3 & 41.2 & 50.0 & 88 & & & & & & \\
\hline $172-1-42-62$ & gectegggetgegttactteg & 21 & 64.0 & -30.0 & 40.3 & 66.7 & 85 & 85 & & & & & \\
\hline $1 F 3-1 ; 53-73$ & egttacttogttgoggatagg & 21 & 56.6 & -26.3 & 41.4 & 52.4 & 87 & 87 & & & & & \\
\hline 174 & geggataggactateteggtg & 21 & 57.3 & -26.3 & 37.2 & 57.1 & 85 & 83 & & & & & \\
\hline 175 & tetcggtgttttetgactge 20 & 55.4 & -25.0 & 36.3 & 50.0 & 76 & 73 & & & & & & \\
\hline 176 & tggettcogogagtcattge 20 & 61.1 & -27.9 & 43.4 & 60.0 & 92 & 92 & & & & & & \\
\hline $1 R_{1}-1-348-368$ & atgtgggtttgecttacaagc & 21 & 56.7 & -26.1 & 40.2 & 47.6 & 92 & 90 & & & & & \\
\hline $1 R 2-1{ }^{2}-264-286$ & 5 tgttggttacttctcggtaaggt & 23 & 55.3 & -27.4 & 38.8 & 43.5 & 80 & 80 & & & & & \\
\hline 1R3_1_ $161-180$ & atgatgccetcatgtcogta 20 & 55.5 & -25.1 & 37.7 & 50.0 & 82 & 82 & & & & & & \\
\hline 1R4__-136-157 & tgttttatcgacacettcgtcc & 22 & 55.2 & -26.6 & 42.8 & 45.5 & 85 & 80 & & & & & \\
\hline \multirow{2}{*}{\multicolumn{3}{|c|}{$\begin{array}{c}\text { Forward_PrimerID Sequence }\left(5^{\prime}-3^{\prime}\right) \text { Tm( }{ }^{\circ} \text { PCR_Fragment_Size (bp) } \\
\text { Topt }\left({ }^{\circ} \mathrm{C}\right)\end{array}$}} & \multicolumn{3}{|c|}{ Primer_Quality (\$) } & \multicolumn{3}{|c|}{ Reverse_PrimerID } & \multirow{2}{*}{\multicolumn{3}{|c|}{ Sequence $\left(5^{\prime}-3^{\prime}\right)$}} & $\operatorname{Im}\left({ }^{\circ} \mathrm{C}\right)$ & Primer_Quality ( $($ ) \\
\hline & & & & \multirow{2}{*}{\multicolumn{2}{|c|}{ 1R1_1_348-36 }} & \multirow{2}{*}{\multicolumn{4}{|c|}{8 atgtgggtttgecttacaagc }} & & & & \\
\hline $171 \_1 \_18-3 \overline{9}$ & tegtattcaggegtacettetg & 56.5 & 88 & & & & & & & 56.7 & 90 & 351 & 62.0 \\
\hline $171_{-} 1_{-} 18-39$ & tegtattcaggegtacettetg & 56.5 & 88 & \multirow{2}{*}{\multicolumn{2}{|c|}{$\begin{array}{l}1 R 2-1-264-286 \\
1 R 3-1-161-180\end{array}$}} & \multirow{2}{*}{\multicolumn{4}{|c|}{$\begin{array}{l}6 \text { tgttggttacttctcggtaaggt } \\
0 \text { atgatgecetcatgtcogta } 55.5\end{array}$}} & 55.3 & 80 & 269 & 61.0 \\
\hline $1 \mathrm{~F}_{1} 1-18-39$ & tegtattcaggegtacettetg & 56.5 & 88 & & & & & & & 82 & 163 & 61.0 & \\
\hline $1 F 1$ & tegtattcaggegtacettetg & 56.5 & 88 & \multicolumn{2}{|c|}{$1 R 4{ }_{1}-136-15 ?$} & & 55.2 & 80 & 140 & 60.0 \\
\hline $1 F 3$ & egttacttcgttgeggatagg & 56.6 & 87 & \multirow{2}{*}{\multicolumn{2}{|c|}{$1 R 1-1-348-368$}} & \multicolumn{4}{|c|}{ atgtgggtttgecttacaagc } & 56.7 & 90 & 316 & 62.0 \\
\hline $1 F 3$ & egttacttegttgeggatagg & 56.6 & 87 & & & \multirow{2}{*}{\multicolumn{4}{|c|}{ tgttggttacttctcggtaaggt }} & 55.3 & 80 & 234 & 61.0 \\
\hline $1 F 3-1-53-73$ & egttacttegttgeggatagg & 56.6 & 87 & $13_{3}$ & $161-180$ & 0 atgatg & & & & 82 & 128 & 60.0 & \\
\hline $1 F 3-1-53-73$ & cgttacttegttgeggatagg & 56.6 & 87 & 1R4_- & $136-157$ & 7 tgttet & categace & accttegt & & 55.2 & 80 & 105 & 60.0 \\
\hline $174-1-65-85$ & geggataggactateteggtg & 57.3 & 83 & 1R1_- & $-348-368$ & 8 atgtgg & ggtttgec & ettacaag & & 56.7 & 90 & 304 & 62.0 \\
\hline 174 -1_65-85 & geggataggactateteggtg & 57.3 & 83 & $1 R_{-}^{1}$ & $264-286$ & 6 tgttgg & jttacttc & eteggtaa & agt & 55.3 & 80 & 222 & 61.0 \\
\hline example2 & & & & & & & & & & & & & \\
\hline $2 \mathrm{~F}_{1} 1{ }_{1}-9-28$ & gageacataggaacgeteca 20 & 57.3 & -25.8 & 44.5 & 55.0 & 92 & 92 & & & & & & \\
\hline $2 \mathrm{~F}_{2}-1-58-77$ & tautcgcggcacacggaggt 20 & 60.8 & -28.1 & 47.6 & 60.0 & 84 & 80 & & & & & & \\
\hline $2 \mathrm{~F}_{3}-1-84-105$ & gtgetaacaaccacggtaagtc & 22 & 56.8 & -27.0 & 40.6 & 50.0 & 88 & 88 & & & & & \\
\hline 2F4_- $96-115$ & acggtaagtetcacgtecas 20 & 55.3 & -25.2 & 40.3 & 50.0 & 82 & 80 & & & & & & \\
\hline $2 R 1$ & ttggacgtgagacttacegt 20 & 55.3 & -25.2 & 37.6 & 50.0 & 82 & 82 & & & & & & \\
\hline $2 R_{2}{ }_{1}-84-105$ & gacttaccgtggttgttagcac & 22 & 56.8 & -27.0 & 38.3 & 50.0 & 88 & 86 & & & & & \\
\hline $2 R 3-67-88$ & agcacatatttacetecgtgtg & 22 & 55. & -26.4 & 41.8 & 45.5 & 90 & 80 & & & & & \\
\hline $2 R A_{-1}^{-1} 56-75$ & etcegtgtgecgegattagg 20 & 61.7 & -27.9 & 46.4 & 65.0 & 87 & 87 & & & & & & \\
\hline $2 R 5{ }_{1} 1{ }_{-16-35}$ & egagetetggagogttecta 20 & 58.8 & -26.6 & 42.0 & 60.0 & 84 & 84 & & & & & & \\
\hline $2 R_{6}{ }_{1}-5-25$ & agcgttcetatgtgetcegca & 21 & 60.7 & -28.9 & 46.1 & 57.1 & 87 & 87 & & & & & \\
\hline Forward_Primer & IID Sequence $\left(5^{\prime}-3^{\prime}\right)$ & $\operatorname{Im}\left({ }^{\circ} \mathrm{C}\right)$ & Primer & Qualit & cy (\$) & Revers & se_Prime & erID & Sequet & nce $\left(5^{\prime}-\right.$ & & $\operatorname{Im}\left({ }^{\circ} \mathrm{C}\right)$ & Primer_Quality (3) \\
\hline $\begin{array}{l}\text { PCR_Fra } \\
2 \mathrm{~F} 1 \\
\end{array}$ & agment_Size (bp) Top & & & & & & & & & & & & \\
\hline 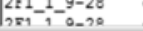 & 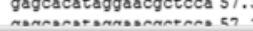 & ${ }_{02}^{92}$ & $201-1-9$ & & 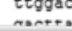 & & & & 56. & 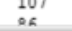 & 07.0 & a) $n$ & \\
\hline
\end{tabular}

Fig. 3 Primer design result window

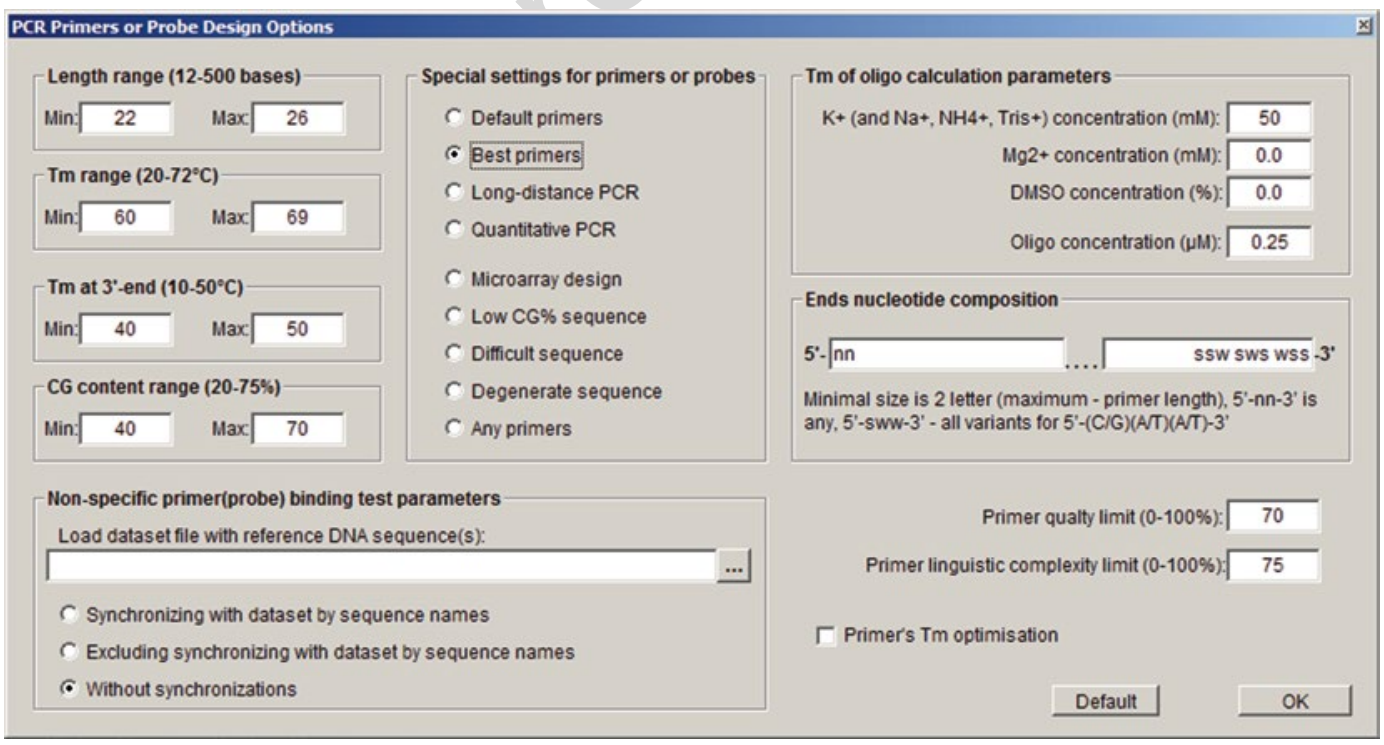

Fig. 4 The "PCR Primers or Probes Design Options" window 


\section{Author's Proof}

PCR Primer and Probe Design and Oligonucleotide Assembly and Analysis

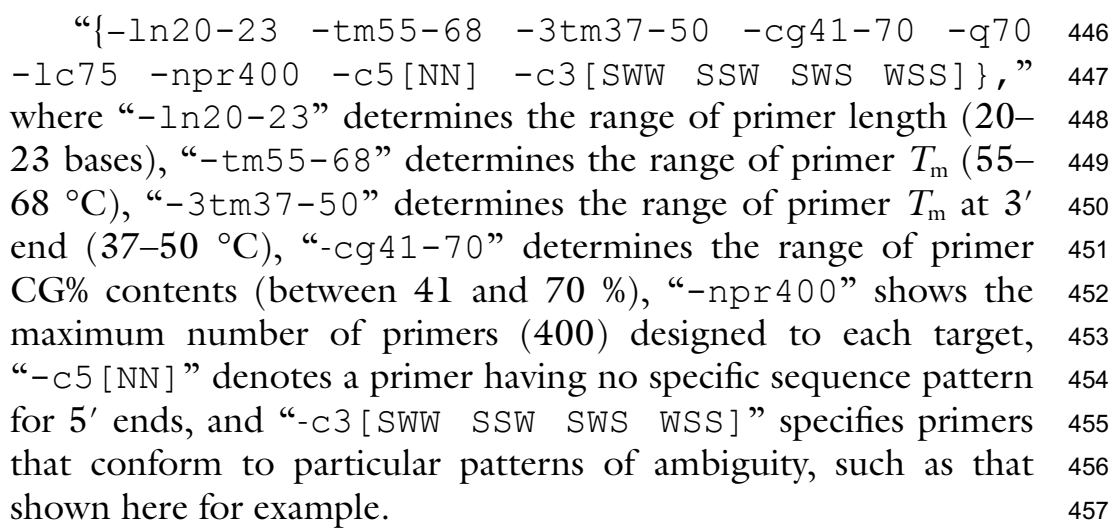

5.3 Examples for Primer Selection Region

Users can specify, individually for each sequence, multiple locations for both forward and reverse primer placement with the commands: “-EpdN1-N2" for forward primers and “-RpdN1-N2" for reverse primers, where from N1 to N2 are bases from the selected regions; “-pdN1-N2" (see more at: http://primerdigital.com/soft/pcr_ help.html). Alternatively, users can specify multiple locations for both forward and reverse primers using [ "and"] inside each sequence: the software allows multiple and independent locations for both forward and reverse primers inside each of the sequences. Whilst PCR primer design will be performed independently for different targets, multiplex PCR primer design can be performed simultaneously with multiple amplicons within a single sequence as well as for different sequences, i.e., all possible combinations of ["and"] inside one or more sequences. By default, the software designs primers within the entire sequence length. Optionally, users can specify, individually for each sequence, multiple locations for both forward and reverse primers with the commands:

1. The same location for both forward and reverse primers will be designed in the central [nnnnnnnnnn] part ("[]" is used only once):

$\ldots \ldots[n n n n n n] \ldots$

2. Different locations for forward and reverse primers; forward primers will be chosen inside the " $[1 n n n n n n]$ " location and reverse primers inside " $[2 \mathrm{nnnnnn}]$ " location (twice " [] ”): $\ldots \ldots[1 n n n n n n] \ldots[2 n n n n n n] \ldots .$.

3. Primers must flank the central "] nnnnnn ["; forward primers will be chosen from 1 to " $\mathrm{A}]$ " bases and reverse primers will be chosen from base " $[C$ " to the end of sequence: ...... $]$ nnnnnn [C....

4. Forward and reverse primers have an overlapping part "[nnnnnn]"; forward primers will be chosen from "[A to n]" bases and reverse primers will be chosen from " $[n$ base to $C]$ ": $\ldots \ldots[$. . . . . [nnnnnn ] ....C] ...

458

459

460

461

462

463

464

465

466

467

468

469

470

471

472

473

474

475

476

477

478

479

480

481

482

483

484

485

486

487

488

489

490 


\section{Author's Proof}

Ruslan Kalendar et al.

The software allows the selection of any number of independent PCR primer (or probe) designing tasks for each sequence using multiple combinations of " $[\ldots]$ ” and -FpdN1-N2, -RpdN1-N2, or -pdN1-N2 commands. Multiplex PCR can be carried out simultaneously within a single sequence with multiple tasks as well as for different sequences with multiple tasks or a combination of both.

All possible combinations of "[]" (forward) with "[]" (reverse) within the sequence(s):

1. [ ]

2. ] [

3. [ ] [ ]

4. [ [ ] ]

5. ([] []])n or/and ([ []])n.

5.4 User-Defined PCR Product Size

\subsection{PCR Set-up}

Examples with Individual Commands
The PCR product size can be specified in a similar way, with the command: " $(\mathrm{N} 1-\mathrm{N} 2)$ "; these values can be specified in the form of minimum and maximum values for the product size. For example, the " $(400-500)$ " line defines that the product size ranges from 400 to 500 base pairs. In case a user wants to specify a fixed product size, the command should be a single number, for example, “ (500)." FastPCR is flexible and allows PCR product sizes from 50 to 10,000 base pairs in length.

1. Where primers have already been designed, FastPCR can be used to predict the optimal annealing temperature and PCR product length for one or more predesigned primers (with the "-npd" command, which prohibits the primer design). For example, the command:

\section{"-fpr [ggagagtagcttacctcgct cggtaaggttct-tcatgc]} -npd"

will analyze the selected sequence between the two primers $\left(5^{\prime}\right.$ ggagagtagcttacctcgct and 5' cggtaaggttcttcatgc).

2. Design primers with a difference in $T_{\mathrm{m}}$ of about $10^{\circ}$, e.g., for LATE-PCR:

"-Ftm50-55 -Rtm64-68 -pTMs10."

3. Design primers with a specific restriction enzyme site at the $3^{\prime}$ end ("-z3eNameEn zyme," “-Fz 3eNameEnzyme," “-Rz3e NameEnzyme," where "NameEnzyme" is the name of the restriction enzyme: "-z3eXceI." The alternative command ( "-C3NN") is also used for a special primer location. For example, “-c3YCATGR" is the same as "-z3eXceI": both commands will design primers with the recognition site for Xce I endonucleases $5^{\prime}-\mathrm{YCATGR}-3^{\prime}$ at the $3^{\prime}$ end of the primers.

4. Additional bases can be added to primer ends using the commands " $-5 e N N$ " or " $-3 e N N$," where 5 or 3 denotes the end at 


\section{Author's Proof}

which to add the extra bases and "NN" is a given sequence of one to more bases. For example, "-F5eCGACG -R5eTTTTTT," means adding the sequence "CGACG" to forward primers and “TTTTTT" to reverse primers, both at the $5^{\prime}$ ends.

5.6 BisulphiteModified DNA

5.7 Uniqueness of Primers

\subsection{PCR Primer Design}

The "C $\gg \mathrm{T}$ bisulphite conversion" option allows the design of 538 specific PCR primers for in silico bisulphite conversion for both 539 strands. Only cytosine not followed by guanidine (CpG 540 methylation) will be replaced by thymine:

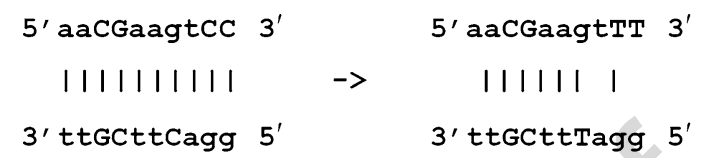

Optionally, the user can synchronize the primer test for secondary, nonspecific binding with a dataset of sequence names. The program recognizes that a given sequence in the screening library dataset (from loading the dataset file) is the same as the sequence for which it is designing primers; this allows sequence selection to be made even if the selection matches the screening sequence perfectly. This allows the same dataset to be used for both primer design and screening without having to make a new screening database for each sequence. In other words, a dataset that contains sequences $\mathrm{A}, \mathrm{B}, \mathrm{C}$, and $\mathrm{D}$ can be used both for choosing primers and for checking primer specificity. Alternatively, the user can input preexisting primers into a second "Additional sequence(s) or pre-designed primers (probes) list" text editor. These primers or probes will be checked for compatibility (inter-primer-dimer formation) with newly designed primers. The number of preexisting primers is not limited to one or two; it can be as many as the user needs.

The PCR primer design algorithm generates a set of primers having 560 a high likelihood of success in virtually any amplification protocol. All PCR primers designed by FastPCR can be used for PCR or sequencing experiments. The program is able to generate either long oligonucleotides or PCR primers for the amplification of gene-specific DNA fragments of user-defined length. FastPCR provides a flexible approach to designing primers for many applications and for both linear and circular sequences. It will check if either primers or probes have secondary binding sites in the input sequences that may give rise to additional PCR products. The selection of the optimal target region for the design of long oligonucleotides is performed in the same way as for PCR primers. The basic parameters in primer design are also used to measure the oligonucleotide quality and to evaluate the thermodynamic stability of the $3^{\prime}$ and $5^{\prime}$ terminal bases. 


\section{Author's Proof}

Ruslan Kalendar et al.

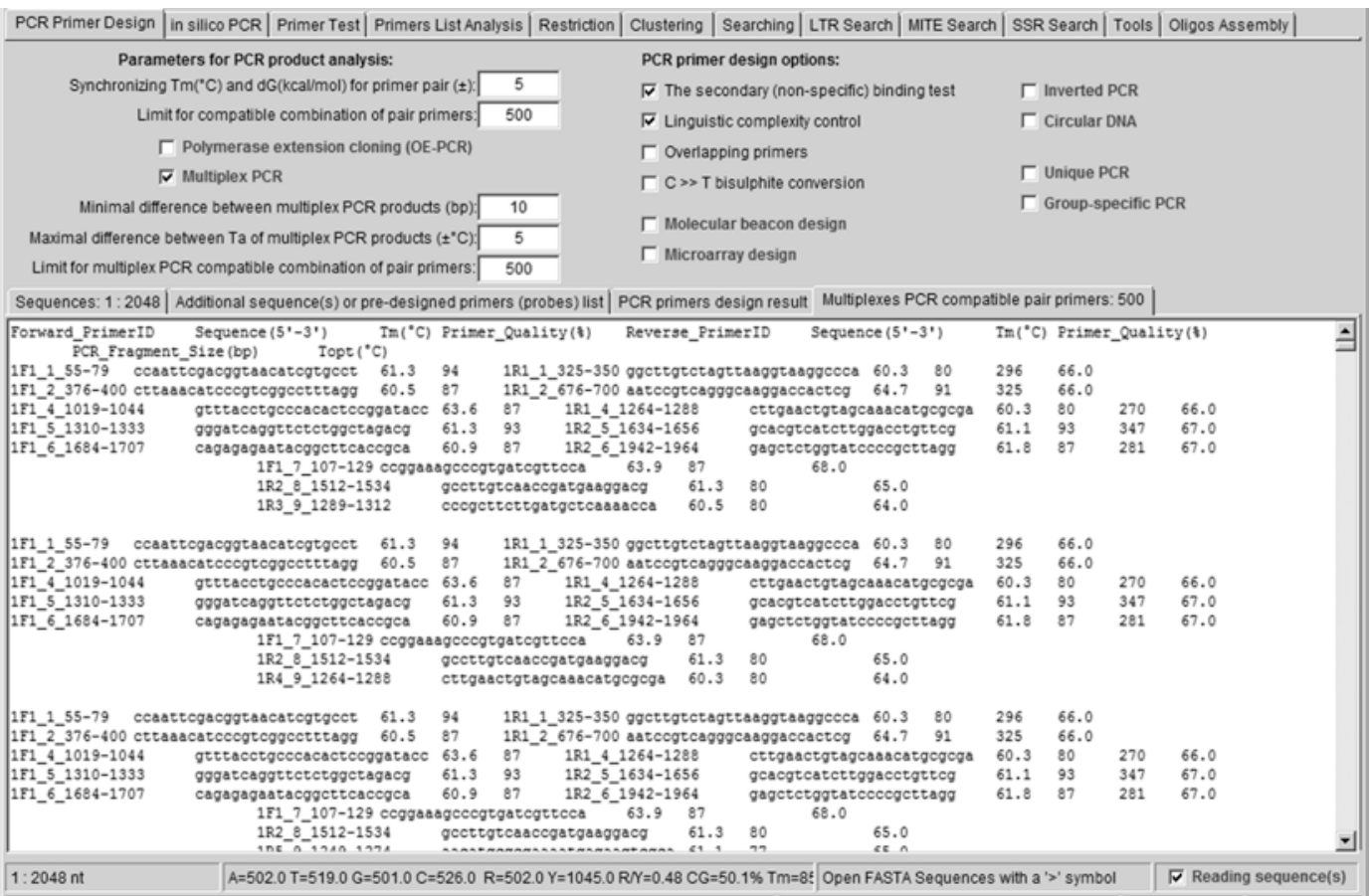

Fig. 5 An example of multiplex PCR result

Both the proposal of primer pairs and the selection of the best pairs are possible. The user can vary the product size or design primer pairs for the whole sequence without specifying parameters by using default or preset parameters. Preset parameters are specified for various situations: for example, for sequences with low CG content, for long-distance PCR, for degenerate sequences, or for manual input. A list of the best primer candidates and all compatible primer pairs that are optimal for PCR is generated. Users can specify, individually for each sequence, multiple locations for both forward and reverse primers within each sequence, whilst PCR design will be performed independently for different targets. Primers for multiplex PCRs can be designed from a single or from multiple targets (Fig. 5).

The program generates primer pairs (and probes) from the input sequences and shows the optimal annealing temperature for each primer pair and the sizes of PCR products together with information for each designed primer. Suggested primers and primer pairs are produced in tabulated format for MS Excel or Open Office (Table 3). The spreadsheets show the following properties: the automatically generated primer name, primer sequence, sequence location, direction, length, melting temperature, CG content (\%), molecular weight, molar extinction coefficient, linguistic complexity (\%), and PQ. For compatible primer pairs, the annealing temperature and PCR product size are also provided. 


\section{Author's Proof}

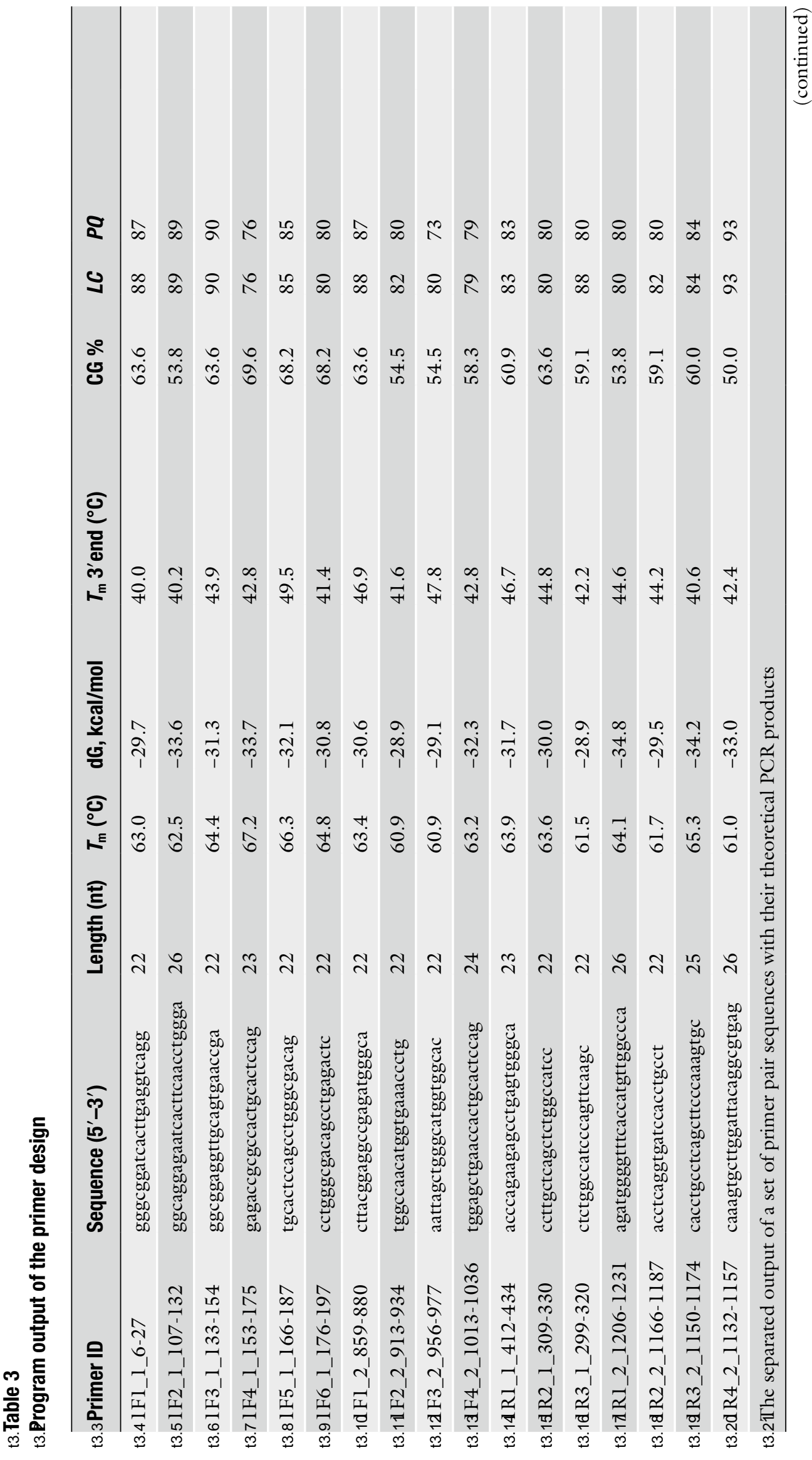




\section{Author's Proof}

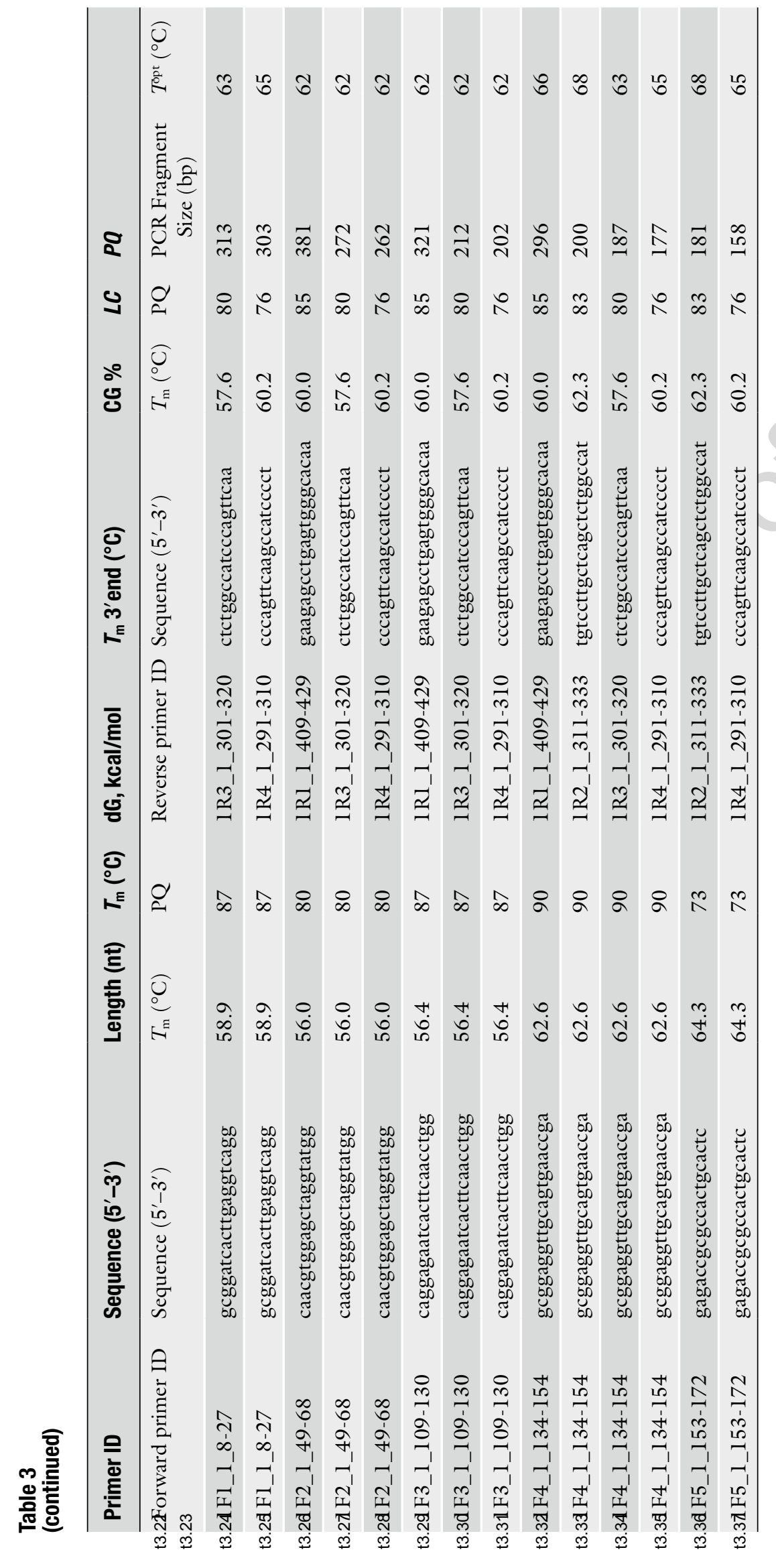

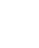$$
\text { . }
$$ 


\section{Author's Proof}

\subsection{Multiplex PCR Primer Design}

Multiplex PCR is an approach commonly used to amplify several DNA target regions in a single reaction. The simultaneous amplification of many targets reduces the number of reactions that needs to be performed; multiplex PCR thus increases throughput efficiency. The design of multiplex PCR assays can be difficult because it involves extensive computational analyses of primer pairs for interactions. The multiplex PCR algorithm is based on the fast non-recursion method, with the software performing checks on product size and primers' thermodynamics parameters (enthalpy$\mathbf{d H}$ and Gibb's free energy- $\mathbf{d G}$ ) compatibility and cross-dimer formation for all primers. To achieve uniform amplification of the targets, the primers must be designed to bind with equal efficiencies to their targets. FastPCR can quickly design a set of multiplex PCR primers for all input sequences and/or multiplex targets within each sequence. PCR conditions may need to be adjusted, for example, by increasing or decreasing the annealing temperature so that all products are amplified equally efficiently.

To achieve uniform amplification, most existing multiplex primer design packages use primer melting temperature. In practical terms, the design of almost identical $T_{\mathrm{a}} \mathrm{s}$ and $T_{\mathrm{m}} \mathrm{s}$ is very important. The melting temperatures of the PCR products are also important because these are related to annealing temperature values. The $T_{\mathrm{m}}$ of a PCR product directly depends on its CG content and its length; short products are more efficiently amplified at low PCR annealing temperatures (100 bp, 50-55 $\left.{ }^{\circ} \mathrm{C}\right)$ than are long products $\left(>3,000 \mathrm{bp}, 65-72{ }^{\circ} \mathrm{C}\right)$. For most multiplex PCRs, there is usually a small variation (up to $5{ }^{\circ} \mathrm{C}$ ) between the optimal $T_{2} \mathrm{~s}$ of all primer pairs. The annealing temperature must be optimal in order to maximize the likelihood of amplifying the target genomic sequences whilst minimizing the risk of nonspecific amplification. Further improvements can be achieved by selecting the optimal set of primers that maximize the range of common $T_{\mathrm{m}} \mathrm{s}$. Once prompted, FastPCR calculates multiplex PCR primer pairs for given target sequences. The speed of calculation depends on the numbers of target sequences and primer pairs involved.

An alternative way to design compatible multiplex PCR primer pairs is to use predesigned primers as references for the design of new primers. The user can select input options for the PCR products such as the minimum product size differences between the amplicons. Primer design conditions can be set individually for each given sequence or all primers can be designed using the same values; selected settings have higher priority for PCR primer or probe design than the general settings. The results include primers for individual sequences, compatible primers, product sizes, and annealing temperatures. Because clear differentiation of the products is dependent on using compatible primer pairs in the single reactions, the program recovers all potential variants of primer combinations for analyses of the chosen DNA regions and provides, 


\section{Author's Proof}

Ruslan Kalendar et al.

in tabular form, their compatibility including information on primer-dimers, cross-hybridization, product size overlaps, and similar alternative primer pairs based on $T_{\mathrm{m}}$. The user may choose those alternative compatible primer pair combinations that provide the desired product sizes. Using the program, researchers can select predesigned primer pairs from a target for their desired types of PCRs by changing the filtering conditions as mentioned above. For example, a conventional multiplex PCR requires differently sized (at least by $10 \mathrm{bp}$ ) amplicons for a set of target genes, so the value for the minimum size difference between PCR products can be selected.

In addition to avoid amplifying different amplicons of the same size, multiplex PCR must also minimize the generation of primerdimers and secondary products, which becomes more difficult with increasing numbers of primers in a reaction. To avoid the problem of nonspecific amplification, FastPCR selects primer pairs that give the most likelihood of producing only the amplicons of the target sequences by choosing sequences which avoid repeats or other motifs. The program allows the user to design not only compatible pairs of primers but also compatible single primers for different targets or sequences. The input data can be either a single sequence with a minimum two internal tasks or many sequences with or without internal tasks. Most of the parameters on the interface are self-explanatory. Optionally, the user is asked to provide the sequence and select oligonucleotide designing parameters.

On the PCR Primer Design tab, the user clicks on the Multiplex PCR option. The user then selects the limit for the number of primer pairs (the default is 100), the minimal size difference between multiplex PCR products (the default $10 \mathrm{bp}$ ), and the maximal difference between the $T_{\mathrm{a}} \mathrm{s}$ of the PCR products (the default is $\pm 5^{\circ} \mathrm{C}$ ). After specifying inputs and primer design options, the user can execute the primer design task. Once the design of the primer set is completed, the result will appear in two Result text editors: PCR primer design result and Multiplex PCR compatible pair primers. Figure 8 shows the access to the PCR primer design output. The result text editor PCR primer design result will display the individual PCR primer design data, including the primer list and the compatible primer pairs for all the sequences and their internal tasks. The second Multiplex PCR compatible pair primers text editor collects final search results that are presented as a list of the sets of the compatible primer pairs for multiplex PCR.

5.10 Group-Specific PCR Primers
Group-specific amplification, also called family-specific and sequence-specific amplification, is an important tool for comparative studies of related genes, sequences, and genomes that can be applied to studies of evolution, especially for gene families and for cloning new related sequences. Specific targets such as homologous genes 
or members of a transposable element family can be amplified to uncover DNA polymorphisms associated with these sequences or 694 other genetic investigations. The overall strategy of designing group-specific PCR primers uses a hash index of 12 -mers to identify common regions in the target sequences, followed by standard PCR primer design for the current sequence, and then testing of complementarity of these primers to the other sequences. FastPCR performs sequences multiple alignment or does accept alignment sequences input, giving it the flexibility to use a different strategy for primer design. If required, it can design degenerate PCR primers to amplify the polymorphic region of all related sequences.

The FastPCR package designs large sets of universal primer pairs for each given sequence, identifies conserved regions, and generates suitable primers for all given targets. The steps of the algorithm are performed automatically and the user can influence the settings for the primer design options. The quality of primer design is dependent on sequence relationships, genetic similarity, and suitability of the consensus sequence for the design of good primers. The software is able to generate group-specific primers for each set of sequences independently, which are suitable for all sequences. Primer alignment parameters for group-specific PCR primers are similar to those used for in silico PCR. The user chooses Group-specific PCR on the PCR Primer Design tab. After specifying inputs and PCR primer design options, the user can execute the PCR primer design task. The program takes multiple separate DNA sequences either in FASTA or at alignment formats.

Once the primer set design is complete, the result will appear in Result text editor, as the PCR primer design result. Figure 6 shows the access to PCR primer design output (Table 3 ). The result text editor PCR primer design result displays the individual group-specific PCR primer design data, including the primer list and compatible primer pairs for all the sequences and their internal tasks where suitable primers are found. In the case where an alignment has been input, the result text editor displays only one group-specific PCR primer design set, including degenerate primers, in the primer list as well compatible primer pairs for all the sequences.

5.11 Simple Sequence Repeat Locus Search and PCR Primer Design
SSRs or microsatellites are short tandem repeats of one or more bases. Microsatellites are ubiquitously distributed throughout eukaryotic genomes, often highly polymorphic in length, and thereby an important class of markers for population genetic studies. Our approach to locating SSRs is to analyze low-complexity regions in DNA by using linguistic sequence complexity. This method allows the detection of perfect and imperfect SSRs with a single, up to 10-base, repeat motif. Each entry sequence is processed for identification of SSRs and the SSR flanking regions are used to design compatible forward and reverse primers for their amplification by PCR.
731 732 733 734 735 736 737 738 739 740 741 


\section{Author's Proof}

Ruslan Kalendar et al.

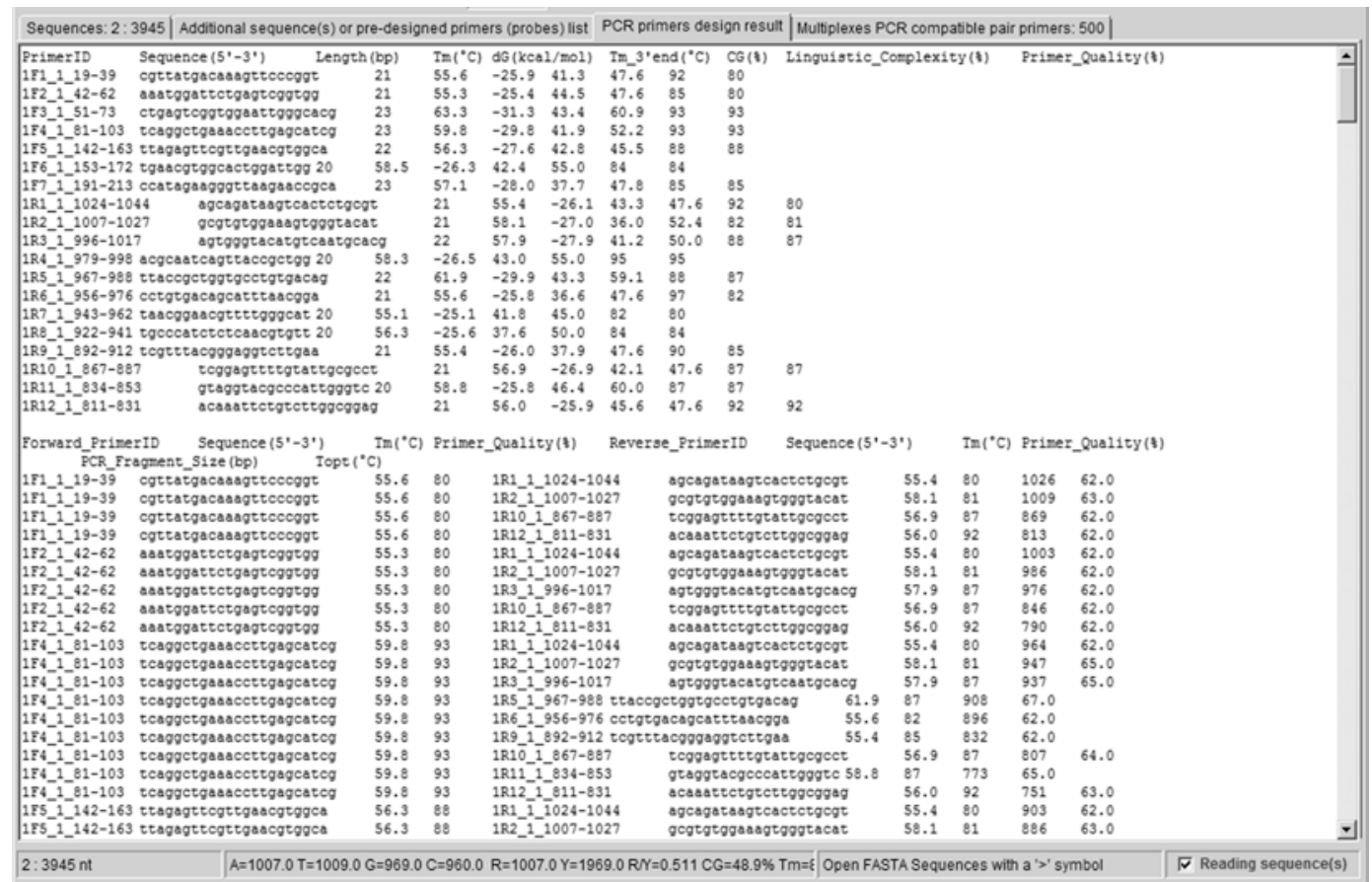

Fig. 6 An example of group-specific PCR result

FastPCR identifies all SSRs within each entry sequence and designs compatible PCR primer pairs for each SSR locus. The default PCR primer design parameters are that the primers must be within 100 bases from either side of the identified SSR. Often the sequences available around SSR loci are not suitable for designing good primers; the user can increase or decrease the distance from either side to find more efficient and compatible primer pairs. The capabilities of FastPCR make it a complete bioinformatics tool for the use of microsatellites as markers, from discovery through to primer design. For example, the user can specify PCR primer design to SSR loci within 200 bp around an SSR, with the command: “-ssr/200." The software finds all SSR sites and then will design PCR primers and compatible primer pairs independently for each SSR locus.

5.12 Oligonucleotide Design for In Vitro Long Sequence Synthesis
The application to make long synthetic DNA molecules relies on the in vitro assembly of a set of short oligonucleotides, either for the LCR [37] or for assembly PCR [38]. These oligonucleotides should be adjacent on the same strand and overlap the complementary oligonucleotides from the second strand. There are two major parameters for designing oligonucleotides for gene synthesis for LCR or assembly PCR. First, the oligonucleotides 
should share similar $T_{\mathrm{m}}$ values. Second, a given oligonucleotide sequence should be unique to avoid multiple nonspecific binding that may lead to incorrect assembly. The software must dynamically choose the length of the oligonucleotides to ensure both the specificity and the uniform $T_{\mathrm{m}}$. The algorithm of FastPCR is able to design oligonucleotides for long sequences containing repeats and to minimize their potential nonspecific hybridization during $3^{\prime}$ end extension in PCR. For long sequence assembly, oligonucleotide design starts from the $5^{\prime}$ end of a given sequence; the oligonucleotide length is dynamically changed until a unique $3^{\prime}$ end has been found and the $T_{\mathrm{m}}$ of the oligonucleotide has reached the $T_{\mathrm{m}}$ threshold. All oligonucleotides are designed without gaps between them. The other strand is used for the design of the overlapping oligonucleotides using the same algorithm as above but with the $T_{\mathrm{m}}$ of the overlapping regions reaching the $T_{\mathrm{m}}-15{ }^{\circ} \mathrm{C}$ threshold. The composition of the sequence at the $3^{\prime}$ terminus is important because stability at the $3^{\prime}$ end of the double-stranded complexes will improve the specificity of extension by the polymerase. To reduce nonspecific polymerase extension (and ligation), the algorithm chooses only unique sequences for the $3^{\prime}$ terminus. Minimally, the last two nucleotides at the $3^{\prime}$ terminus must not be complementary to any nonspecific targets. Other complementary regions are less important for assembling multiple fragments by PCR and ligation.

The input data can comprise either a single or many sequences. Most of the parameters on the interface are self-explanatory. The user is asked to provide the sequence and select oligonucleotide designing parameters. The user clicks on Oligo options on the Oligos Assembly tab, and chooses the minimal oligonucleotide length and $T_{\mathrm{m}}$ threshold, which by default are $40 \mathrm{nt}$ and $60{ }^{\circ} \mathrm{C}$, respectively. The interface allows changing $T_{\mathrm{m}}$ calculation parameters. The search process runs after pressing $\mathbf{F 5}$ or from menu bar or toolbox. The research result is presented as a list of oligonucleotides for both strands. On each strand, all oligonucleotides are adjacent with no gap between neighboring primers. An oligonucleotide will overlap two oligonucleotides from the complementary strand. The algorithm pays attention to avoid nonspecific oligonucleotide hybridization to repeated regions. Where it is not possible to design primers outside of repeated sequences, it is likewise difficult to find short specific oligonucleotides. The solution to this problem is to divide the sequence into short segments, design a set of oligonucleotides for each segment independently, and then combine all these segments in the second PCR for final amplification.

5.13 Polymerase Extension PCR for Fragment Assembly
Sequence-independent cloning, including ligation-independent 807 cloning, requires generation of complementary single-stranded 808 overhangs in both the vector and insertion fragments. Similarly, 809 


\section{Author's Proof}

Ruslan Kalendar et al.

multiple fragments can be joined or concatenated in an ordered manner using overlapping primers in PCR. Annealing of the complementary regions between different targets in the primer overlaps allows the polymerase to synthesize a contiguous fragment containing the target sequences during thermal cycling, a process called "overlap extension PCR" (OE-PCR) (Chapter 8) [39]. The efficiency depends on the $T_{\mathrm{m}}$ and on the length and uniqueness of the overlap. To achieve this, the program designs compatible forward and reverse primers at the ends of each fragment, and then extends the $5^{\prime}$ end of primers using sequences from the primers of the fragment that will be adjacent in the final product. The input sequence can be made of either a single or many sequences. The user needs to pay special attention to the preparation of the given sequences for assembly.

Users can specify the locations for both forward and reverse primers design using " [ ]" to bracket the region. The bracketed sequences will be used by the program for designing the overlapping primers. The program selects the overlapping area so that the primers from overlapping fragments are similar in size with optimal annealing temperatures. The program adds the required bases so that the $T_{\mathrm{m}}$ of the overlap is similar to, or higher than, the $T_{\mathrm{m}}$ of the initial primers. Primers are tested for dimer formation within the appropriate primer pairs. The user chooses Polymerase extension cloning (OE-PCR) on the PCR Primer Design tab and selects the limit for multiple-PCR-compatible combinations of pair primers (default is 100). After specifying sequence inputs and PCR primer design options, the user can execute the search task. Once the design of the primer sets is complete, the result will appear in two text editors: PCR primer design result and PCR fragments assembling compatible pair primers. The text editor PCR primer design result window displays the individual PCR primer design data, including the primer list and the compatible primer pairs for all sequences whose primers are found. The PCR fragments assembling compatible pair primers text editor collects the final search result and presents it as a list of sets of compatible primer pairs for individual fragment amplification and assembly. Figure 7 shows a sample result visualization window.

\subsection{In Silico PCR}

Modelling the hybridization of primers to targeted annealing sites is the only way to predict PCR products $[7,24,40-44]$. The last 10-12 bases at the $3^{\prime}$ end of primers are important for binding stability; single mismatches can reduce PCR efficiency, the effect increasing with proximity to the $3^{\prime}$ terminus. FastPCR allows simultaneous testing of single primers or a set of primers designed for multiplex PCR. It performs a fast, gapless alignment to test the complementarity of the primers to the target sequences. For in silico PCR, a quick alignment to detect primer locations on the reference sequence is performed by analyses of both strands using 


\section{Author's Proof}

PCR Primer and Probe Design and Oligonucleotide Assembly and Analysis

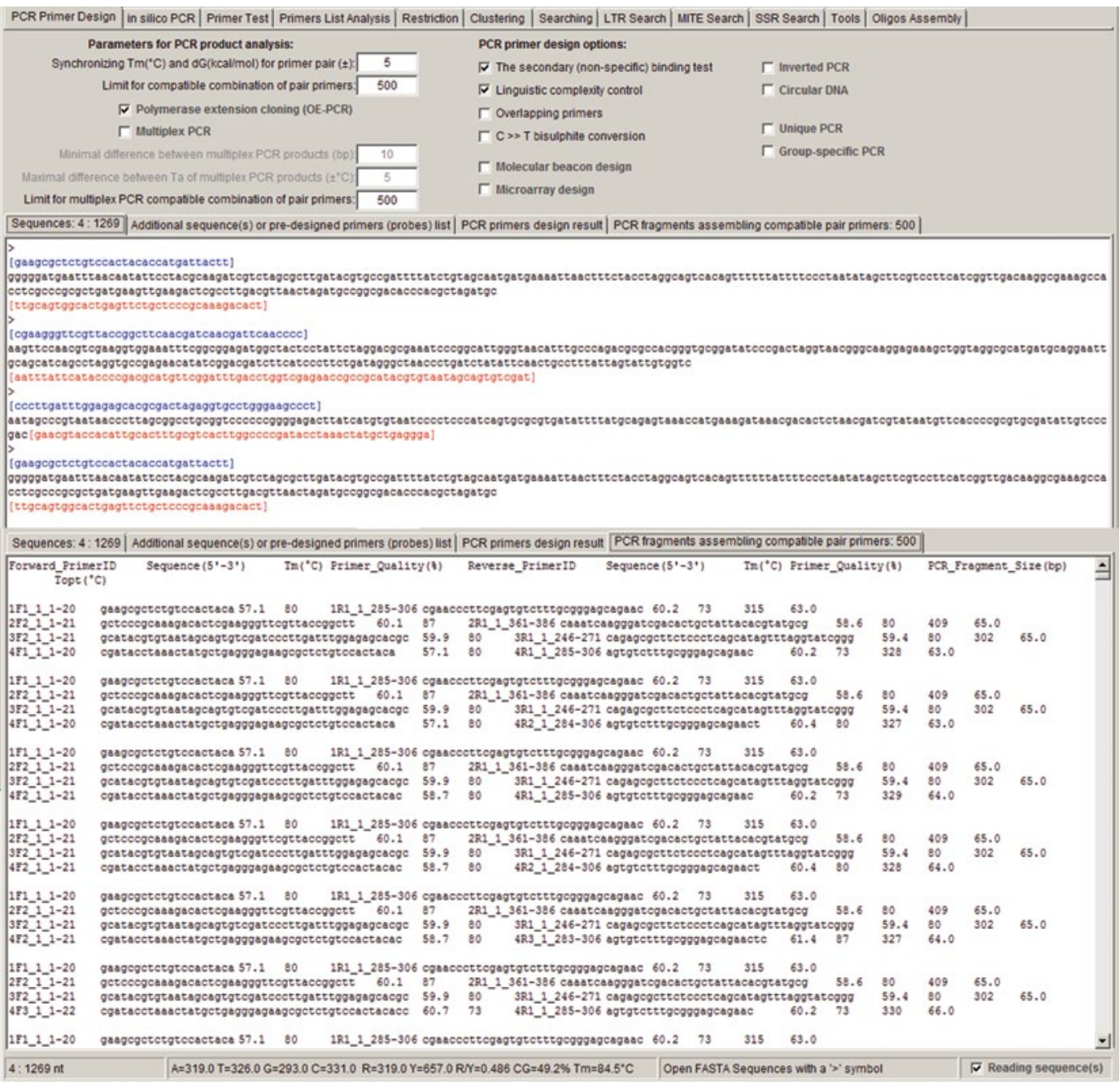

Fig. 7 An example of polymerase extension PCR for fragment assembly result

a hash index of 7- to 12-mers (allowing up to one mismatch) and by calculating the local similarity for the whole primer. The 858 parameters can be altered to allow different degrees of mismatches 859 at the $3^{\prime}$ end of the primers. The parameters for quick alignment 860 may be set: the minimum is $\mathbf{0}-\mathbf{5}$ mismatches (default 2 mismatches) 861 at $3^{\prime}$ end of primer. The program can also handle degenerate 862 primers or probes, including those with $5^{\prime}$ or $3^{\prime}$ tail sequences. It 863 includes the detection of non-Watson-Crick base-pairing in in 864 silico PCR, e.g., the stable guanine mismatches G.G, G.T, and 865 $\mathrm{G} \cdot \mathrm{A}$. Probable PCR products can be found for both linear and 866 circular templates in both standard and inverse PCR, as well as in 867 multiplex PCR and using bisulphite-treated DNA. This in silico 868 tool is useful for quickly analyzing primers or probes against target 869 


\section{Author's Proof}

Ruslan Kalendar et al.

sequences, for determining primer location, orientation, and efficiency of binding, and for calculating primer $T_{\mathrm{m}}$ and annealing temperature in PCR.

The user must input a preexisting primer list into a second Additional sequence(s) or pre-designed primers (probes) list text editor. The number of preexisting primers is not limited; it can be as many as the user needs. The target sequences can be entered either as multiple separate DNA sequences or by opening files from the selected folders. For in silico PCR against whole genome(s) or a list of chromosomes, the user must specify the directory containing the input. The program will be consistent: it will look at each file to find the position of the primers. The user can execute the search task with F5 on the in silico PCR tab or can specify search options including stringency and PCR product detection settings. For the stringency options, users can specify the number of mismatches that the primers are allowed at $3^{\prime}$ terminus. The default specificity settings allow a maximum two mismatches within the $3^{\prime}$ end region of the primers. These mismatches within the $3^{\prime}$ end of the primers should not be located close to each other. Once the primer set design is complete, the results will appear in the text editors In silico PCR Result.

In silico PCR Result text editor reports the specificity of the primers (locations, including target position, similarity, and $T_{\mathrm{m}}$ ), a summary of primer pairs in relation to the PCR template, and detailed information on each primer pair, including its length and $T_{a}$. It will show the target-specific primers that have been found. The actual targets will be listed along with detailed alignments between primers and targets (Fig. 8).

\section{Primer Analyses}

Individual and sets of primers are evaluated using FastPCR or the online software. They calculate primer $T_{\mathrm{m}} \mathrm{s}$ using default or other formulae for features of the primers including normal and degenerate nucleotide combinations, CG content, extinction coefficient, unit conversion (nmol per OD), mass ( $\mu$ g per OD), molecular weight, and linguistic complexity and consider primer PCR efficiency. Users can select either DNA or RNA primers (online: PrimerAnalyser, http://primerdigital.com/tools/ PrimerAnalyser.html) with normal or degenerate oligonucleotides or modifications with various labels (for example, inosine, uridine, or fluorescent dyes). Tools allow the choice of other nearest neighbor thermodynamic parameters or non-thermodynamic $T_{\mathrm{m}}$ calculation formulae.

For LNA modifications the four symbols, $\mathrm{dA}=\mathrm{E}, \mathrm{dC}=\mathrm{F}$, $\mathrm{dG}=\mathrm{J}$, and $\mathrm{dT}=\mathrm{L}$, are used. Both programs perform analyses on- 


\section{Author's Proof}

PCR Primer and Probe Design and Oligonucleotide Assembly and Analysis

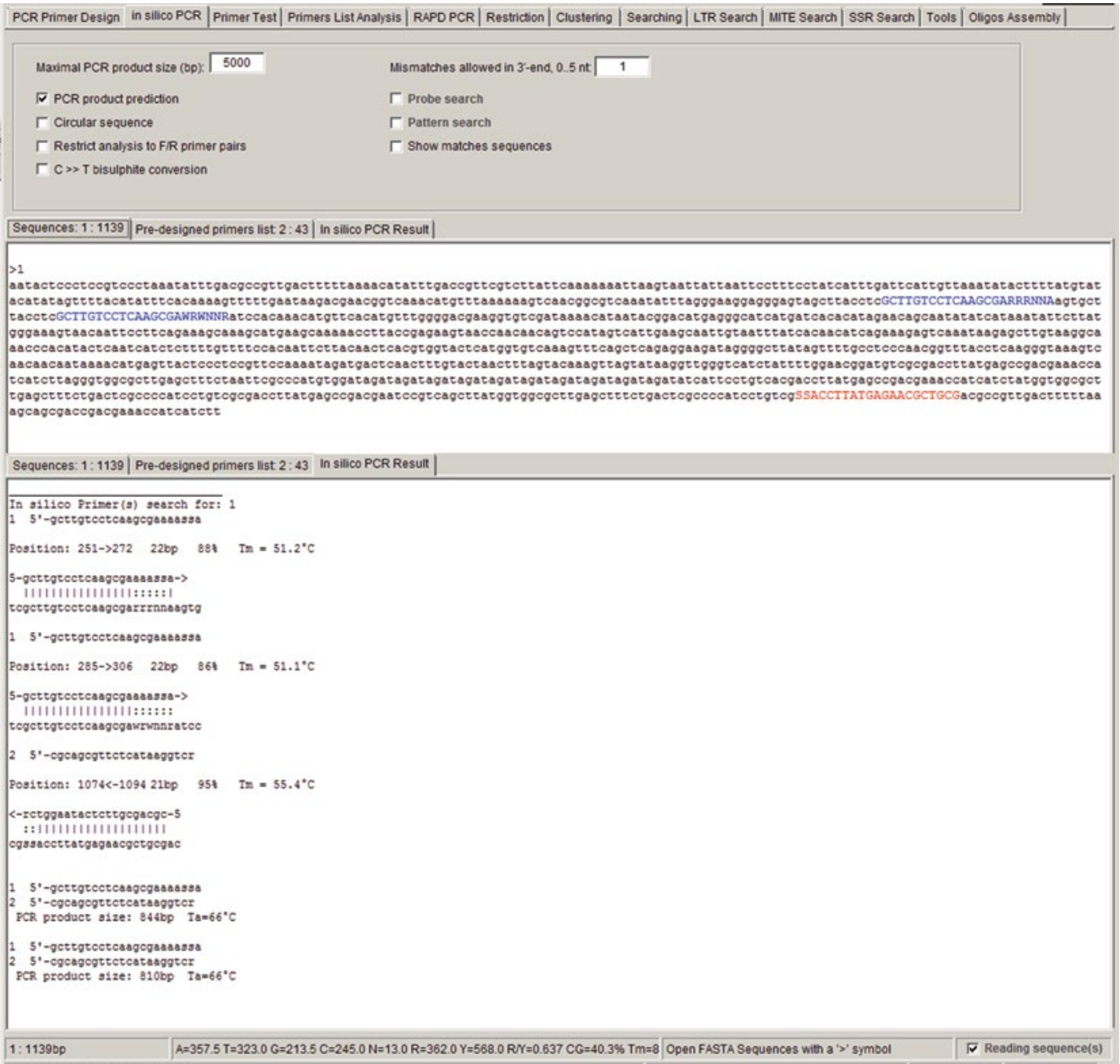

Fig. 8 An example of in silico PCR result

type, allowing users to see the results immediately on screen. They 914 can also calculate the volume of solvent required to attain a specific 915 concentration from the known mass (mg), OD, or moles of 916 oligonucleotide.

All primers are analyzed for intra- and inter-primer interactions regarding formation of dimers. Primer(s) can efficiently hybridize using the $5^{\prime}$ end or the middle of the oligonucleotides. Even though such interactions are not efficiently extended by DNA polymerase, their formation reduces the effective primer concentration available for binding to the targets and their presence can strongly inhibit PCR because double-stranded DNA at high concentrations is a strong inhibitor of DNA polymerase (Fig. 9). 


\section{Author's Proof}

Ruslan Kalendar et al.

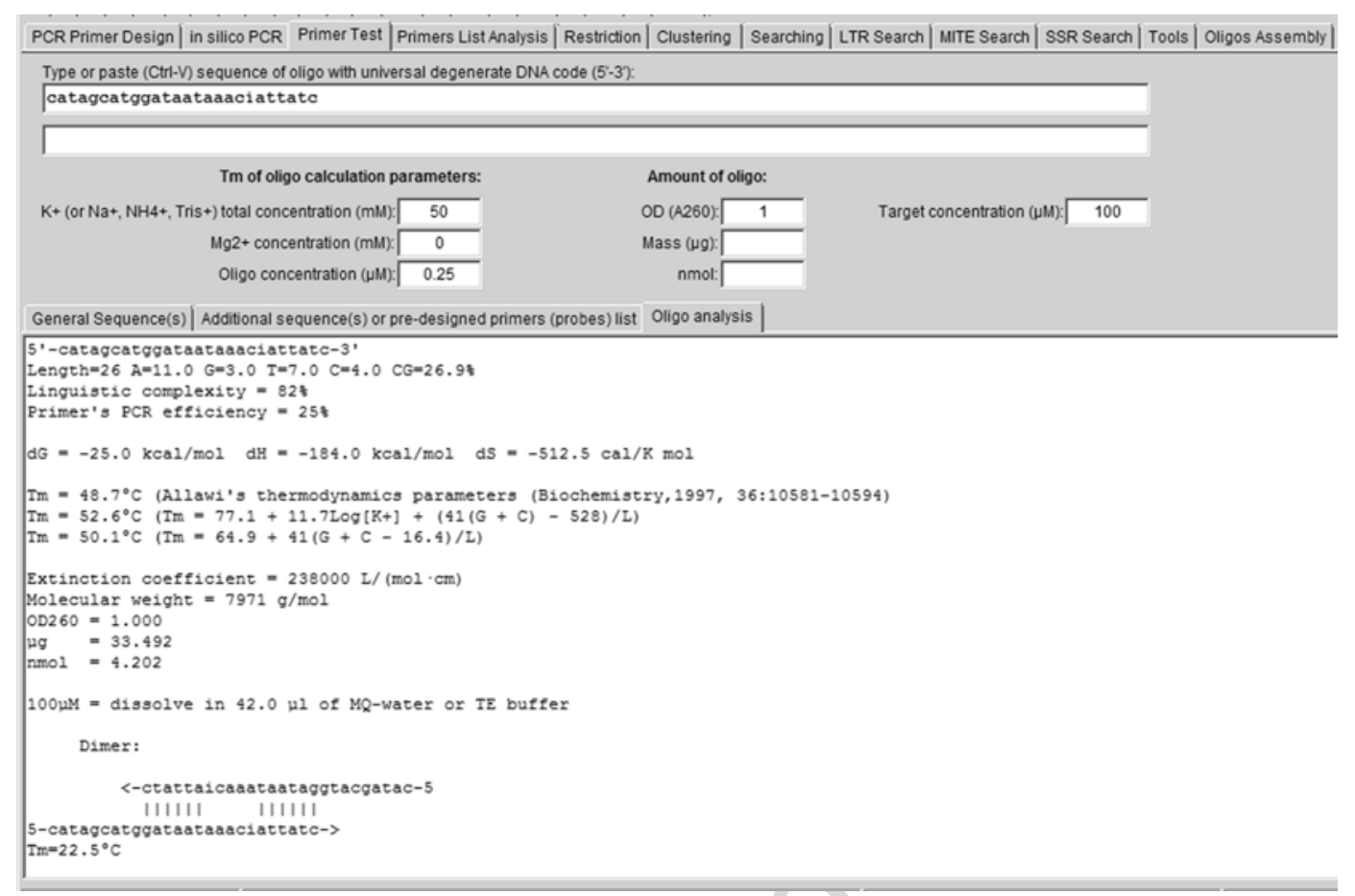

Fig. 9 Example result of the oligonucleotide analysis

\section{Availability}

The FastPCR software is available for download at http:// primerdigital.com/fastpcr.html; the online version is available at http://primerdigital.com/tools/pcr.html. The program manual, licence agreement, and installation files can be found at http:// primerdigital.com/fastpcr/. YouTube tutorial videos have been placed at http://www.youtube.com/user/primerdigital. Web tools are accessible at http://primerdigital.com/tools/.

\section{Acknowledgments}

Web tools are available free to academic institutions, provided that they are used for noncommercial research and education only. They may not be reproduced or distributed for commercial use. This work was partially supported by the companies PrimerDigital Ltd. and Oligomer Ltd. and by the Academy of Finland, Project 134079 . 


\section{References}

1. Untergasser A et al (2012) Primer3-new capabilities and interfaces. Nucleic Acids Res 40:e115. doi:10.1093/nar/gks596

2. Kalendar R, Lee D, Schulman AH (2009) FastPCR software for PCR primer and probe design and repeat search. Genes, Genomes and Genomics 3:1-14

3. Kalendar R, Lee D, Schulman AH (2011) Java web tools for PCR, in silico PCR, and oligonucleotide assembly and analysis. Genomics 98:137-144

4. Marshall OJ (2004) PerlPrimer: crossplatform, graphical primer design for standard, bisulphite and real-time PCR. Bioinformatics 20:2471-2472

5. Owczarzy R et al (2008) IDT SciTools: a suite for analysis and design of nucleic acid oligomers. Nucleic Acids Res 36:W163-9. doi:10.1093/nar/gkn198

6. Bekaert M, Teeling EC (2008) UniPrime: a workflow-based platform for improved universal primer design. Nucleic Acids Res 36:e56. doi:10.1093/nar/gkn191

7. Ye J et al (2012) Primer-BLAST: a tool to design target-specific primers for polymerase chain reaction. BMC Bioinformatics 13:134. doi:10.1186/1471-2105-13-134

8. Giegerich R, Meyer F, Schleiermacher C (1996) GeneFisher-software support for the detection of postulated genes. Proc Int Conf Intell Syst Mol Biol 4:68-77

9. Gadberry MD et al (2005) Primaclade-a flexible tool to find conserved PCR primers across multiple species. Bioinformatics 21:12631264. doi:10.1093/bioinformatics/btil34

10. National Center for Biotechnology Information, National Library of Medicine, Building 38A, Bethesda, MD, 20894. http:// blast.ncbi.nlm.nih.gov/blastcgihelp.shtml

11. Nomenclature for incompletely specified bases in nucleic acid sequences (1984) http://www. chem.qmul.ac.uk/iubmb/misc/naseq.html

12. Allawi HT, SantaLucia J Jr (1997) Thermodynamics and NMR of internal G.T mismatches in DNA. Biochemistry 36:1058110594. doi:10.1021/bi962590c

13. SantaLucia J (1998) A unified view of polymer, dumbbell, and oligonucleotide DNA nearest-neighbor thermodynamics. Proc Natl Acad Sci USA 95:1460-1465

14. Le Novere N (2001) MELTING, computing the melting temperature of nucleic acid duplex. Bioinformatics 17:1226-1227. doi:10.1093/ bioinformatics/17.12.1226
15. Bolton ET, McCarthy BJ (1962) A general method for the isolation of RNA complementary to DNA. Proc Natl Acad Sci USA 48: 1390-1397

16. Guedin A et al (2010) How long is too long? Effects of loop size on G-quadruplex stability. Nucleic Acids Res 38:7858-68. doi:10.1093/ nar/gkq639

17. Wallace RB et al (1979) Hybridization of synthetic oligodeoxyribonucleotides to $\Phi \mathrm{X} 174$ DNA: the effect of single base pair mismatch. Nucleic Acids Res 6:3543-57. doi:10.1093/ nar/6.11.3543

18. von Ahsen N, Wittwer CT, Schutz E (2001) Oligonucleotide melting temperatures under PCR conditions: nearest-neighbor corrections for $\mathrm{Mg}^{2+}$, deoxynucleotide triphosphate, and dimethyl sulfoxide concentrations with comparison to alternative empirical formulas. Clin Chem 47:1956-1961

19. Kypr J et al (2009) Circular dichroism and conformational polymorphism of DNA. Nucleic Acids Res 37:1713-25. doi:10.1093/ nar/gkp026

20. Gabrielian A, Bolshoy A (1999) Sequence complexity and DNA curvature. Comput Chem 23:263-74. doi:10.1016/S0097-8485 (99)00007-8

21. Orlov YL, Potapov VN (2004) Complexity: an internet resource for analysis of DNA sequence complexity. Nucleic Acids Res 32:W628-33. doi:10.1093/nar/gkh466

22. Gilson MK et al (1997) The statisticalthermodynamic basis for computation of binding affinities: a critical review. Biophys J 72:1047-69. doi:10.1016/S0006-3495(97) 78756-3

23. Peyret $\mathrm{N}$ et al (1999) Nearest-neighbor thermodynamics and NMR of DNA sequences with internal A.A, C.C, G.G, and T.T mismatches. Biochemistry 38:3468-3477. doi:10.1021/bi9825091

24. Watkins NE Jr, SantaLucia J Jr (2005) Nearestneighbor thermodynamics of deoxyinosine pairs in DNA duplexes. Nucleic Acids Res 33:6258-67. doi:10.1093/nar/gki918

25. Sen D, Gilbert W (1992) Guanine quartet structures. Methods Enzymol 211:191-199

26. Il'icheva IA, Florent'ev VL (1992) Fourstranded complexes of oligonucleotidesquadruplexes. Mol Biol (Mosk) 26:512-531

27. Shing HP (1994) The non-B-DNA structure of $\mathrm{d}(\mathrm{CA} / \mathrm{TG}) \mathrm{n}$ does not differ from that of Z-DNA. Proc Natl Acad Sci USA 91:9549-9553 


\section{Author's Proof}

Ruslan Kalendar et al.

1050

1051

1052

1053

1054

1055

1056

1057

1058

1059

1060

1061

1062

1063

1064

1065

1066

1067

1068

1069

1070

1071

1072

1073

1074

1075

1076

1077

1078

1079

1080

1081

1082
28. SantaLucia J Jr, Hicks D (2004) The thermodynamics of DNA structural motifs. Annu Rev Biophys Biomol Struct 33:415-40. doi:10.1146/ annurev.biophys.32.110601.141800

29. Williams JGK et al (1990) DNA polymorphisms amplified by arbitrary primers are useful as genetic-markers. Nucleic Acids Res 18:6531-5. doi:10.1093/nar/18.22.6531

30. Welsh J, Mcclelland M (1990) Fingerprinting genomes using PCR with arbitrary primers. Nucleic Acids Res 18:7213-8. doi:10.1093/ nar/18.24.7213

31. Kalendar R, Schulman A (2006) IRAP and REMAP for retrotransposon-based genotyping and fingerprinting. Nat Protoc 1:2478-84. doi:10.1038/nprot.2006.377

32. Chang RY, O'Donoughue LS, Bureau TE (2001) Inter-MITE polymorphisms (IMP): a high throughput transposon-based genome mapping and fingerprinting approach. Theor Appl Genet 102:773-781

33. Nelson DL et al (1989) Alu polymerase chain reaction: a method for rapid isolation of human-specific sequences from complex DNA sources. Proc Natl Acad Sci USA 86: 6686-6690

34. Sinnett D et al (1990) Alumorphs-human DNA polymorphisms detected by polymerase chain reaction using Alu-specific primers. Genomics 7:331-334

35. Jurka J et al (2005) Repbase update, a database of eukaryotic repetitive elements. Cytogenet Genom Res 110:462-7. doi:10.1159/000084979
36. TREP, the Triticeae Repeat Sequence Database (2008) http://wheat.pw.usda.gov/ITMI/ Repeats/

37. Landegren U et al (1988) A ligase-mediated gene detection technique. Science 241:1077-1080

38. Higasa K, Hayashi K (2002) Ordered catenation of sequence-tagged sites and multiplexed SNP genotyping by sequencing. Nucleic Acids Res 30:E11

39. Quan J, Tian J (2009) Circular polymerase extension cloning of complex gene libraries and pathways. PLoS One 4:e6441. doi:10.1371/journal.pone.0006441

40. Cao YF et al (2005) Information theory-based algorithm for in silico prediction of PCR products with whole genomic sequences as templates. BMC Bioinformatics 6:190. doi:10.1186/ 1471-2105-6-190

41. Rubin E, Levy AA (1996) A mathematical model and a computerized simulation of PCR using complex templates. Nucleic Acids Res 24:3538-45. doi:10.1093/nar/24.18.3538

42. Lexa M, Valle G (2003) PRIMEX: rapid identification of oligonucleotide matches in whole genomes. Bioinformatics 19:2486-2488

43. Nishigaki K et al (2000) Whole genome sequence-enabled prediction of sequences performed for random PCR products of Escherichia coli. Nucleic Acids Res 28:1879-1884

44. Rotmistrovsky K, Jang W, Schuler GD (2004) A web server for performing electronic PCR. 1113 Nucleic Acids Res 32:W108-12. doi:10.1093/ 1114 nar/gkh450 


\section{Author's Proof}

\section{Author Queries}

Chapter No.: 18

0002067381

\begin{tabular}{|l|l|l|}
\hline Queries & Details Required & Author's Response \\
\hline AU1 & $\begin{array}{l}\text { Please check if the edit made in the sentence "It calculates the melting ... resuspen- } \\
\text { sion calculator." is appropriate. }\end{array}$ & \\
\hline AU2 & $\begin{array}{l}\text { Please check if the edit made in the sentence "Getting started with a ... to type." is } \\
\text { appropriate. }\end{array}$ & $\begin{array}{l}\text { Please check if the edit made in the sentence "Primer pairs are analyzed ... melting } \\
\text { temperatures." is appropriate. }\end{array}$ \\
\hline AU3 & $\begin{array}{l}\text { Please check if the edit made in the sentence "Multiplex PCR can be ... of both." is } \\
\text { appropriate. }\end{array}$ & \\
\hline AU4 & $\begin{array}{l}\text { Please provide closing parenthesis in sentence "Design primers with a specific restric- } \\
\text { tion ..." }\end{array}$ & \\
\hline AU5 & $\begin{array}{l}\text { Please check if the edit made in the sentence "The program takes multiple ... align- } \\
\text { ment formats." is appropriate. }\end{array}$ & \\
\hline AU6
\end{tabular}

